\title{
Supersymmetric Rényi entropy and defect operators
}

\author{
Tatsuma Nishioka $^{a}$ and Itamar Yaakov ${ }^{b}$ \\ ${ }^{a}$ Department of Physics, Faculty of Science, The University of Tokyo, \\ Bunkyo-ku, Tokyo 113-0033, Japan \\ ${ }^{b}$ Kavli IPMU (WPI), UTIAS, The University of Tokyo, \\ Kashiwa, Chiba 277-8583, Japan \\ E-mail: nishioka@hep-th.phys.s.u-tokyo.ac.jp, itamar.yaakov@ipmu.jp
}

ABSTRACT: We describe the defect operator interpretation of the supersymmetric Rényi entropies of superconformal field theories in three, four and five dimensions. The operators involved are supersymmetric codimension-two defects in an auxiliary $\mathbb{Z}_{n}$ gauge theory coupled to $n$ copies of the SCFT. We compute the exact expectation values of such operators using localization, and compare the results to the supersymmetric Rényi entropy. The agreement between the two implies a relationship between the partition function on a squashed sphere and the one on a round sphere in the presence of defects.

KEYWORDS: Nonperturbative Effects, Supersymmetric Gauge Theory, Supersymmetry and Duality

ARXIV EPRINT: 1612.02894 


\section{Contents}

1 Introduction 1

1.1 Supersymmetric Rényi entropy and defects 2

1.2 Rényi entropy and discrete gauge theories 3

2 Supersymmetric defects from supersymmetric $\mathbb{Z}_{n}$ gauge theory 4

2.1 Supersymmetric $\mathbb{Z}_{n}$ gauge theory 5

2.2 The $3 d \mathcal{N}=2$ codimension-two defect 5

2.3 The $4 d \mathcal{N}=2$ codimension-two defect 6

2.3.1 The $\mathcal{N}=2$ calculation on the four-sphere 7

2.3.2 Introducing the defect 8

$\begin{array}{lll}2.3 .3 & \text { The supersymmetric Lagrange multiplier } & 9\end{array}$

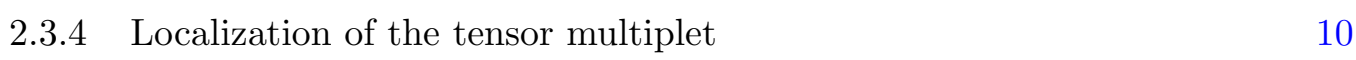

2.3.5 Modification of the matrix model 11

2.4 The $5 d \mathcal{N}=1$ codimension-two defect 13

$\begin{array}{lll}2.4 .1 & \text { Killing spinors on the five-sphere } & 13\end{array}$

$\begin{array}{lll}2.4 .2 & \text { Codimension-two defects } & 14\end{array}$

$\begin{array}{lll}2.4 .3 & \text { Defects and supersymmetric Lagrange multiplier } & 15\end{array}$

3 Squashing from defects $\quad 16$

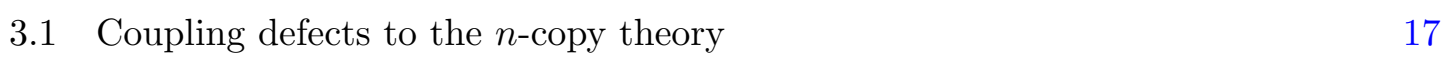

$\begin{array}{ll}3.2 \text { Mass terms and vortices } & 19\end{array}$

$\begin{array}{ll}3.3 & \text { The matrix models } \\ & 21\end{array}$

3.4 The scalar moduli space and classical contributions 22

$\begin{array}{lll}3.5 & \text { Perturbative contributions } 23\end{array}$

3.6 Non-perturbative contributions — instantons and contact-instantons 24

3.6.1 Sewing of instantons 25

3.6.2 Classical and Chern-Simons contributions depending on instantons 26

$\begin{array}{lll}3.6 .3 & \text { Fundamental hypermultiplets fluctuations } & 27\end{array}$

3.6.4 Adjoint hypermultiplet or vector multiplet fluctuations 28

$\begin{array}{ll}3.6 .5 & \text { The third point }\end{array}$

$\begin{array}{ll}3.7 \quad n \rightarrow 1 / n \text { duality } & 30\end{array}$

$\begin{array}{lll}4 & \text { Discussion } & 31\end{array}$

$\begin{array}{ll}\text { A Conventions } & 32\end{array}$

B Special functions $\quad 33$

C The instanton partition function $\quad 34$ 


\section{Introduction}

The non-local nature of quantum entanglement is one of the sharpest characteristics by which quantum physics differentiates itself from classical physics. Entanglement occupies a central position in quantum information theory and, increasingly, in various branches of theoretical physics such as condensed matter and high energy physics. Entangled states are ubiquitous and of particular interest in many-body quantum systems. Rényi entropy is a refined measure of the entanglement a given state possesses when the Hilbert space is split into states supported on a spatial region $\Sigma$ and those supported on its complement. In a local quantum field theory, employing the replica trick [1], the $n^{\text {th }}$ Rényi entropy amounts to

$$
S_{n} \equiv \frac{1}{1-n} \log \left|\frac{Z_{n}}{\left(Z_{1}\right)^{n}}\right|
$$

The partition function $Z_{n}$ is defined on an $n$-fold cover $\mathcal{M}_{n}$ branched over the entangling surface $\partial \Sigma$ of a (Euclidean) manifold on which the theory is placed. The absolute value taken in the definition (1.1) has no effect in unitary theories, but is necessary to incorporate the case of a complex partition function which we will deal with when supersymmetry is implemented on a curved space.

It follows from (1.1) that knowing the partition function $Z_{n}$ is more or less equivalent to calculating Rényi entropies, and there are a few situations where the exact values are known (see e.g. [1-3]). A common practice in handling the conical singularity around $\partial \Sigma$, present in the calculation of $Z_{n}$ for $n>1$, is to smooth out the tip by introducing a regulator, calculate the partition function on the smoothed space, and take the singular limit [4]. This approach is highly advantageous as it reformulates the problem as a calculation in quantum field theory on a curved space.

Another complementary approach is to represent the partition function $Z_{n}$ as a product of correlation functions of twist operators that create the proper monodromies around $\partial \Sigma[1,5]$. Twist operators are codimension-two (non-local in $d>2$ dimensions) objects that specify the boundary conditions on the entangling surface, and the twisting is done for an $n$-fold copy of the original theory. In what follows, we will illustrate the interplay between the two approaches in a particular situation where the exact calculation of $Z_{n}$ is possible: the supersymmetric Rényi entropy computed using localization [6]. We will restrict ourselves to a spherical entangling surface $\partial \Sigma=\mathbb{S}^{d-2}$ in $d$ dimensions, work in the vacuum state of the SCFT, and examine supersymmetric gauge theories of type $3 d \mathcal{N}=2$, $4 d \mathcal{N}=2$, and $5 d \mathcal{N}=1$. The motivation for doing so is twofold. First, it is interesting to compare the two different looking localization calculations and find out how they match. Second, the microscopic definition of the defect operators, ${ }^{1}$ presented in section 2 , could be useful when examining dualities in which the SCFT participates. These could be dualities between different Lagrangian field theories, or holographic dualities between an SCFT and string theory on an appropriate background.

\footnotetext{
${ }^{1}$ Twist operators are a subclass of defect operators. These terms will be used interchangeably in this paper.
} 


\subsection{Supersymmetric Rényi entropy and defects}

The vacuum Rényi entropy is a non-local observable which can be defined for any $d$ dimensional quantum field theory whose Hilbert space can be factorized in a local manner. For a conformal field theory, and for integer Rényi parameter $n$, this observable is equivalent to two different objects, each of which can be defined by a suitable Euclidean path integral:

I. The partition function on an $n$-fold multiple covering of the $d$-sphere $\mathbb{S}^{d}$, branched along $\partial \Sigma$, with appropriate boundary conditions at the branching loci [7].

II. The partition function of an $n$-fold copy of the theory - henceforth referred to as the $n$-copy theory - on $\mathbb{S}^{d}$ with codimension-one defect operators acting between copies (cf. [8]).

The equivalence between these two objects is tautological. The object in II is simply a relabeling of the degrees of freedom, one for each of the $n$ sheets of the branched sphere. The defect operators are defined to reproduce the boundary conditions implied by the original geometry.

One may introduce a linear field redefinition, acting in the $n$-copy theory, to diagonalize the action of the defects, introducing defects which act on just one copy at a time. In the special case of a free theory, the action written using the redefined fields does not couple the copies. The computation in this case is equivalent to a third object:

III. The partition function of an $n$-fold copy of the theory on $\mathbb{S}^{d}$ with codimension-two defect operators acting on each copy - equivalently, the $k^{\text {th }}$ copy is coupled to a background connection with holonomy $e^{2 \pi i \frac{k}{n}}$ around $\partial \Sigma$, with $k \in\{0, \ldots, n-1\} .^{2}$ This is really a product of the $n$ partition functions, since the copies are decoupled.

We have codimension-two defects since the partition function over free fields, which are subject to a monodromy when circling $\partial \Sigma$, does not care about the position of $\Sigma$.

One may also alter the definition of the Rényi entropy to preserve additional symmetries of the theory. Specifically, a subset of superconformal symmetry can be preserved by altering the boundary conditions in the object I, II, or III (equivalently, changing the defects). The resulting observable is called the supersymmetric Rényi entropy [6] (see also [10-18] for further developments). ${ }^{3}$ It has been observed that the result in this case is equivalent to yet another object:

IV. The partition function of a single copy of the theory on a squashed $\mathbb{S}^{d}$, with a suitable supersymmetry preserving action. The squashing parameter, which determines the non-round metric, is related to the Rényi parameter $n$ in a simple way, such that $n=1$ corresponds to the round sphere.

\footnotetext{
${ }^{2}$ The holonomy prescription could be different if the field in question is a fermion, or carries additional global symmetry charges [9]. We will make a specific choice later on.

${ }^{3}$ Some of the $3 d$ theories we consider are not superconformal. However, deformation invariance of the partition function is enough to guarantee that the result can then be interpreted as the supersymmetric Rényi entropy of the SCFT to which the original theory flows [6].
} 
The simplest explanation for the last equivalence is as follows. Preserving supersymmetry means that the Euclidean action is invariant under some supersymmetry transformation $\delta$. It is well known that the path integral in this case is insensitive to $\delta$-exact deformations, either of the action or in the form of $\delta$-exact insertions. ${ }^{4}$ The objects I and IV are related by such a deformation (c.f [19]).

Despite their simplicity, there is something interesting to be said about forms III and IV of the supersymmetric Rényi entropy. The calculation of the path integrals representing either III or IV can be performed exactly using localization. This involves splitting the fields of the theory into an interacting part - the moduli - and a free part - the fluctuations. The latter can be put into the form III. Since the two calculations look quite different, and in some contexts have different interpretations, it is interesting and potentially useful to determine exactly how they give the same result. This will be our primary goal.

\subsection{Rényi entropy and discrete gauge theories}

The setup described above for calculating the Rényi entropy at integer Rényi parameter can be alternatively thought of as introducing a defect in a discrete gauge theory coupled to the $n$-copy theory. ${ }^{5}$ For our purposes, it is sufficient to consider the gauge group $\mathbb{Z}_{n}$ acting by cyclic permutation on the copies, although the $n$-copy theory is invariant under the full permutation group $\mathfrak{S}_{n} .{ }^{6}$ If we choose to think of $\mathbb{Z}_{n}$ as a gauge symmetry, we can reasonably treat the defects which implement the calculation of the Rényi entropy as codimension-two objects. Only operators charged under the $\mathbb{Z}_{n}$ symmetry can detect the position of the codimension-one defect. Gauging $\mathbb{Z}_{n}$ means that all such operators are projected out.

We would now like to incorporate supersymmetry into the definition of the $\mathbb{Z}_{n}$ gauge theory and the defect. A simple way of doing so is to write down a version of the discrete gauge theory which is realized by higher form abelian gauge fields [20]. A BF type theory with one ordinary gauge field $(A)$ and one $(d-2)$-form field $(B)$ works nicely. The reduction to $\mathbb{Z}_{n}$ gauge symmetry is implemented by using $B$ as a Lagrange multiplier. The action for the gauge sector is

$$
S_{\mathrm{BF}}=\frac{i n}{2 \pi} \int F \wedge B
$$

where $F$ is the field strength of $A . S_{\mathrm{BF}}$ is invariant under gauge transformations for both $A$ and $B$ as long as $n$ is an integer.

The codimension-two defect could be realized by taking a flat connection with prescribed holonomy and coupling it to the free fields of the $n$-copy theory. The way to do this in the BF theory is to insert the operator

$$
\exp \left(i k \oint_{\partial \Sigma} B\right)=\exp \left(i k \int B \wedge[\partial \Sigma]\right)
$$

\footnotetext{
${ }^{4}$ There are additional restrictions on the deformation. A $\delta$-exact deformation to the action must be annihilated by $\delta$ and should have a positive semi-definite real part. Specifically, any deformation must be such that it does not alter the convergence properties of the path integral.

${ }^{5}$ The authors are grateful to Daniel Jafferis for suggesting a discrete gauge theory interpretation for our calculation.

${ }^{6}$ The symmetry which shifts the copies by one is known as the replica $\mathbb{Z}_{n}$ symmetry.
} 
where $[\partial \Sigma]$ is the Poincaré dual to the cycle $\partial \Sigma$ representing the entangling surface. This operator is invariant under the $B$ gauge transformations as long as $k$ is an integer. Integrating out $B$ in the theory with the action (1.2), and in the presence of this operator, induces a holonomy for $A$, around $\partial \Sigma$ of strength $e^{2 \pi i \frac{k}{n}}$. After the linear field redefinition, $A$ can serve as the flat connection for the $k^{t h}$ copy for the Rényi parameter $n$. One may then extend the fields $A, B$ and the terms in the action to their supersymmetric versions to create a SUSY-BF theory [21]. ${ }^{7}$

One possible obstruction to using ordinary gauge fields is that the original theory may have its own, possibly non-abelian, gauge fields. It may not be possible to couple the field $A$ to these non-abelian gauge fields in a manner consistent with gauge invariance. Note that the original description of the codimension-one defect already implies that any gauge group of the $n$-copy theory is broken to a diagonal subgroup, acting simultaneously and identically on the copy at either side, at the defect. This type of the reduction of the gauge invariance at the position of a defect is quite common (see e.g. [22]). Moreover, gauge fields often carry supersymmetric moduli which cannot be coupled to the codimension-two defect in the way described above. We will treat all of these problems in an ad hoc manner.

The organization of the paper is as follows. In section 2 we introduce a supersymmetric version of the $\mathbb{Z}_{n}$ gauge theory based on higher form abelian gauge superfields. Component expressions for the superfields and actions are readily available in the literature. We then derive the abelian version of the codimension-two supersymmetric defect operator as a classical supersymmetric configuration for a vector supermultiplet on the round $d$ dimensional sphere. We use this configuration to write down an ordinary supersymmetric Wilson type codimension-two operator for the tensor multiplet, using the supersymmetric version of (1.3), and perform localization in the presence of this operator and the BF term. We then derive the resulting effect on any matrix model to which the original vector multiplet could potentially be coupled. In section 3, we show that the matrix model for the $n$-copy theory on the round $\mathbb{S}^{d}$, after a specific modification by such abelian defects, is equal to the matrix model on the squashed $\mathbb{S}^{d}$, including all classical and one-loop contributions. In the process, we show how the moduli for the squashed sphere can be thought of as $n$ sets of moduli for the round sphere, which are sewn together by the defect. We end with a discussion of possible applications.

\section{Supersymmetric defects from supersymmetric $\mathbb{Z}_{n}$ gauge theory}

In this section, we define the supersymmetric $\mathbb{Z}_{n}$ gauge theory, and supersymmetric codimension-two defects, which we later use to calculate the supersymmetric Rényi entropy. We then calculate the expectation values of these defects using localization. We treat supersymmetric gauge theories of type $3 d \mathcal{N}=2,4 d \mathcal{N}=2$, and $5 d \mathcal{N}=1$.

\footnotetext{
${ }^{7}$ By SUSY-BF, we mean an untwisted supersymmetric version of BF theory of the type considered in [21]. We are not concerned, in this paper, with the topological field theory aspects of supersymmetric BF theories - which is the context in which they were originally introduced. Specifically, we couple the SUSY-BF fields to the $n$-copy theory and introduce localizing terms, both of which can spoil the topological properties.
} 


\subsection{Supersymmetric $\mathbb{Z}_{n}$ gauge theory}

We construct a supersymmetric $\mathbb{Z}_{n}$ gauge theory, and a supersymmetric codimension-two defect, by introducing a pair of dynamical supermultiplets: a vector multiplet $V$ and a $(d-2)$-form multiplet $E$. The field strength for the latter sits in the familiar linear multiplet, denoted by $G$. We introduce the supersymmetric analogues of the terms used in the discrete gauge theory

$$
S_{\mathrm{BF}} \rightarrow \frac{i n}{2 \pi} \int G V,
$$

and

$$
\exp \left(i k \oint_{\partial \Sigma} B\right) \rightarrow \exp \left(i k \oint_{\partial \Sigma} E\right) .
$$

The terms on the right hand side are schematic superspace integrals, for which we write component expressions later. The operator (2.2) can also be written as

$$
\exp \left(\frac{i k}{2 \pi} \int G V_{\text {defect }}\right) \text {, }
$$

where $V_{\text {defect }}$ is a background configuration for a vector multiplet invariant under a subset of the supersymmetries, whose component expression will be worked out below, and the integral is over the entire superspace. Integrating out $G$ results in a supersymmetric delta function setting

$$
V=\frac{k}{n} V_{\text {defect }} .
$$

We call the coefficient

$$
q_{k}^{\text {vortex }}=\frac{k}{n},
$$

the vortex charge.

\subsection{The $3 d \mathcal{N}=2$ codimension-two defect}

In three dimensions, both $V$ and $E$ are ordinary vector multiplets. The superfield $G$ is the field strength superfield which can be used to write the Yang-Mills term in $3 d .{ }^{8}$ The codimension-two defect is a vortex loop of the type examined, for instance, in [23]. Its supersymmetric version was analyzed in $[24,25]$. The calculation of the supersymmetric Rényi entropy in three dimensions using defects was carried out in [6]. We briefly review it below.

The localization calculation for an $\mathcal{N}=2$ theory on the round $\mathbb{S}^{3}$ reduces the path integral to an integration over a single Lie algebra valued scalar: the constant mode

$$
\sigma=-D
$$

of the real fields $\sigma$ and $D$ appearing in the $\mathcal{N}=2$ vector multiplet [26, 27]. The resulting matrix model expression for the partition function will be given in section 3.3. Specifically, the fluctuation determinant for an abelian vector multiplet is 1 .

\footnotetext{
${ }^{8}$ This is a real linear superfield, which is sometimes denoted by $\Sigma$.
} 
A supersymmetric defect which mimics the effects of computing the supersymmetric Rényi entropy can be introduced by considering an additional background abelian vector multiplet

$$
\left\{A_{\mu}, \sigma^{A}, D^{A}, \text { fermions }\right\} .
$$

The defect configuration is of the form

$$
d A=\alpha \star[\gamma]_{D}, \quad \star D=i \alpha[\gamma]_{D} \wedge[\gamma]
$$

where $[\gamma]$ is the volume form on a maximal $\mathbb{S}^{1} \subset \mathbb{S}^{3}$, and $[\gamma]_{D}$ is the Poincare dual to this cycle.

For $\alpha=\frac{k}{n}$, this configuration can be imposed on a dynamical vector multiplet by considering an additional vector multiplet with connection $B$, an off-diagonal Chern-Simons coupling

$$
S_{\mathrm{BF}}^{(3 d)}=\frac{i n}{2 \pi} \int_{\mathbb{S}^{3}}\left(A \wedge d B+\sigma^{A} D^{B}+\sigma^{B} D^{A}+\text { fermions }\right),
$$

and a supersymmetric abelian Wilson loop of the form

$$
W^{(3 d)}(k) \equiv \exp \left[i k \oint_{\gamma}\left(B-i[\gamma] \sigma^{B}\right)\right]
$$

Localization reduces the above terms to ${ }^{9}$

$$
\begin{aligned}
\exp \left(-S_{\mathrm{BF}}^{(3 d)}\right) & \rightarrow \exp \left(2 \pi i n \sigma^{A} \sigma^{B}\right) \\
W^{(3 d)}(k) & \rightarrow \exp \left(-2 \pi k \sigma^{B}\right)
\end{aligned}
$$

Integrating over the new modulus $\sigma^{B}$ then sets

$$
\sigma^{A}=-i \frac{k}{n}
$$

Note that this vev for $\sigma^{A}$ is off the original contour of integration and represents an imaginary mass term for chiral multiplets to which the $A$ vector multiplet is coupled. We will argue later that an imaginary Higgs type mass for a dynamical vector multiplet can also be thought of this way. The imaginary mass is the entire effect of the original defect on the localization computation. By continuity, the same is true for an arbitrary $\alpha$. We will show below that this continues to hold, for appropriate defects, in four and in five dimensions.

\subsection{The $4 d \mathcal{N}=2$ codimension-two defect}

We consider a $4 d \mathcal{N}=2$ theory consisting of vector multiplets and hypermultiplets. We would like to show that the effect of inserting a codimension-two defect is equivalent to the introduction of an imaginary mass term.

\footnotetext{
${ }^{9}$ We universally denote the Lie algebra valued scalar zero mode as $\sigma$.
} 


\subsubsection{The $\mathcal{N}=2$ calculation on the four-sphere}

We begin by reviewing some aspects of the localization calculation for an $\mathcal{N}=2$ theory on the round, and on the branched four-sphere following [10, 28, 29]. We refer the reader to these papers for explicit actions and localizing terms. The authors of [10] considered a smooth resolution of the branched four-sphere, the resolved four-sphere, which is deformation equivalent to it, and on which localization computations can be performed. In this section, we retain an overall scale $\ell$, associated with the size of the four-sphere, which will later be set to 1 .

We use the following coordinates on an $n$-fold covering of $\mathbb{S}^{4}$ (the branched sphere)

$$
\begin{aligned}
d s^{2} & =\ell^{2}\left(d \theta^{2}+n^{2} \sin ^{2} \theta d \tau^{2}+\cos ^{2} \theta d s_{\mathbb{S}_{2}}^{2}\right), \\
d s_{\mathbb{S}_{2}}^{2} & =d \phi^{2}+\sin ^{2} \phi d \chi^{2} .
\end{aligned}
$$

We use the vielbein

$$
e^{1}=\ell d \theta, \quad e^{2}=n \ell \sin \theta d \tau, \quad e^{3}=\ell \cos \theta d \phi, \quad e^{4}=\ell \cos \theta \sin \phi d \chi,
$$

and take a basis for the Clifford algebra

$$
\begin{aligned}
\gamma_{a} & =\left(\begin{array}{cc}
0 & \bar{\sigma}_{a} \\
\sigma_{a} & 0
\end{array}\right), \\
\sigma_{i} \equiv-i \tau_{i}, \quad \bar{\sigma}_{i} & =i \tau_{i}, \quad \sigma_{4}=\bar{\sigma}_{4}=\mathbb{1},
\end{aligned}
$$

where $\tau_{i}$ are the Pauli matrices.

An $\mathcal{N}=2$ supersymmetry is generated by a foursome of Weyl spinors $\xi_{\alpha A}, \bar{\xi}_{\dot{\alpha} A}$, subject to the reality condition [29]

$$
\xi^{\alpha A} \equiv\left(\xi_{\alpha A}\right)^{\dagger}=\epsilon^{\alpha \beta} \epsilon^{A B} \xi_{\beta B}, \quad \bar{\xi}^{A \dot{\alpha}} \equiv\left(\bar{\xi}_{\dot{\alpha} A}\right)^{\dagger}=\epsilon^{\dot{\alpha} \dot{\beta}} \epsilon^{A B} \bar{\xi}_{\dot{\beta} B} .
$$

The subscript $A$ is an $\mathrm{SU}(2)_{R}$ index. Indices $\alpha, \dot{\alpha}$ indicate a spinor transforming as a doublet under the left and right $S U(2)$ factors of $\operatorname{Spin}(4)$. Indices $\alpha, \dot{\alpha}, A$ are raised with $\epsilon^{\alpha \beta}, \epsilon^{\dot{\alpha} \dot{\beta}}, \epsilon^{A B}$ such that $\epsilon^{12}=1$ and $\epsilon_{A B}=-\epsilon^{A B}$.

In order to preserve rigid supersymmetry, one must solve the Killing spinor equation. The relevant Killing spinor equation for the round/branched four-sphere is

$$
\begin{gathered}
\partial_{\mu} \xi_{A}+\frac{1}{8} \omega_{\mu}^{a b}\left(\sigma_{a} \bar{\sigma}_{b}-\sigma_{b} \bar{\sigma}_{a}\right) \xi-i A_{\mu}^{\mathrm{SU}(2)_{R}}{ }_{A}{ }^{B} \xi_{B}=-i \sigma_{\mu} \bar{\xi}_{A}^{\prime}, \\
\partial_{\mu} \bar{\xi}_{A}+\frac{1}{8} \omega_{\mu}^{a b}\left(\bar{\sigma}_{a} \sigma_{b}-\bar{\sigma}_{b} \sigma_{a}\right) \bar{\xi}_{A}-i A_{\mu}^{\mathrm{SU}(2)_{R}}{ }_{A}{ }^{B} \bar{\xi}_{B}=-i \bar{\sigma}_{\mu} \xi_{A}^{\prime},
\end{gathered}
$$

Here, we have introduced an $\mathrm{SU}(2)_{R}$ background connection $A^{\mathrm{SU}(2)_{R}}$, and set all other supergravity background fields besides the metric to zero. The solution is given by

$$
A^{\mathrm{SU}(2)_{R}}=\frac{n-1}{2}\left(\begin{array}{cc}
1 & 0 \\
0 & -1
\end{array}\right) d \tau,
$$


and

$$
\begin{aligned}
& \xi_{\alpha 1}=e^{-\frac{i}{2}(\tau+\chi)}\left(\begin{array}{c}
-\sin \frac{\theta}{2} \cos \frac{\phi}{2} \\
\cos \frac{\theta}{2} \sin \frac{\phi}{2}
\end{array}\right), \quad \xi_{\alpha 2}=e^{\frac{i}{2}(\tau+\chi)}\left(\begin{array}{c}
\cos \frac{\theta}{2} \sin \frac{\phi}{2} \\
\sin \frac{\theta}{2} \cos \frac{\phi}{2}
\end{array}\right), \\
& \bar{\xi}_{1}^{\dot{\alpha}}=e^{-\frac{i}{2}(\tau+\chi)}\left(\begin{array}{l}
-\sin \frac{\theta}{2} \sin \frac{\phi}{2} \\
-\cos \frac{\theta}{2} \cos \frac{\phi}{2}
\end{array}\right), \quad \bar{\xi}_{2}^{\dot{\alpha}}=e^{\frac{i}{2}(\tau+\chi)}\left(\begin{array}{c}
\cos \frac{\theta}{2} \cos \frac{\phi}{2} \\
-\sin \frac{\theta}{2} \sin \frac{\phi}{2}
\end{array}\right) .
\end{aligned}
$$

$\xi^{\prime}$ can be extracted by contracting with $\sigma^{\mu} / \bar{\sigma}^{\mu}$. In order to use the transformation generated by $\xi$ to perform localization, one should impose an additional constraint to ensure that the square of the transformation does not contain scale or $\mathrm{U}(1)_{R}$ transformations

$$
\xi^{\alpha A} \xi_{\alpha A}^{\prime}=0, \quad \bar{\xi}^{\dot{\alpha} A} \bar{\xi}_{\dot{\alpha} A}^{\prime}=0 .
$$

This constraint has been taken into account above.

As shown in [10, 29], one can introduce supergravity backgrounds for the resolved foursphere such that the same Killing spinors are preserved. Moreover, one may use the same localizing term for the vector and hypermultiplets in the presence of defects/squashing as one does in the round sphere case. These localizing terms, which are described in $[28,29]$, imply that all components of a hypermultiplet must vanish on the moduli space. The contour of integration for vector multiplet scalars compatible with the localizing terms is

$$
\phi^{\dagger}=-\bar{\phi}, \quad\left(D^{A B}\right)^{\dagger}=-D_{A B} .
$$

The localizing terms yield the following moduli space of zero modes for a vector multiplet

$$
\phi=\bar{\phi}=-i \frac{\ell}{2} D_{12}=-i \frac{\sigma}{2},
$$

where $\sigma$ is a constant Lie algebra valued scalar. In addition, there are point-like instantons localized at the north pole $(\theta=0, \phi=0)$, and anti-instantons at the south pole $(\theta=$ $0, \phi=\pi / 2)$. The complete matrix model expression for the partition function is given in section 3.3.

\subsubsection{Introducing the defect}

We would like to introduce a surface defect into the computation on the round sphere. The data for a surface defect can be embedded in a background $\mathcal{N}=2$ abelian vector multiplet

$$
\left\{A_{\mu}, \phi, \bar{\phi}, D_{A B}, \lambda_{A}, \bar{\lambda}_{A}\right\}
$$

where $A_{\mu}$ is the gauge field, $\phi$ and $\bar{\phi}$ are complex scalar fields whose relationship depends on the contour of integration, $D_{A B}$ is an $S U(2)_{R}$ triplet of auxiliary scalars, and $\lambda_{A}, \bar{\lambda}_{A}$ independent Weyl fermions which are $S U(2)_{R}$ doublets. We want a supersymmetric defect, on the round four-sphere, supported only on the two-sphere at $\theta=0$. Such a defect would have a field strength

$$
F_{\text {defect }}=\alpha \delta(\theta) d \theta \wedge d \tau
$$


In order to regularize the above configuration, we introduce a background

$$
A_{\text {defect }}=\alpha g_{\epsilon}(\theta) d \tau, \quad \alpha>0,
$$

such that ${ }^{10}$

$$
F_{\text {defect }}=\alpha g_{\epsilon}^{\prime}(\theta) d \theta \wedge d \tau .
$$

$g_{\epsilon}(\theta)$ is a smooth function satisfying

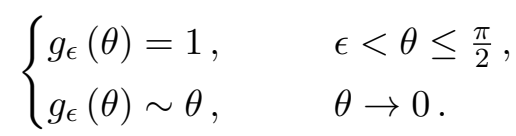

The supersymmetry variation of the gaugino in an abelian vector multiplet is [29]

$$
\begin{aligned}
& \delta \lambda_{A}=\frac{1}{2} \sigma^{\mu \nu} F_{\mu \nu} \xi_{A}+2 \not D \phi \xi_{A}+D_{A B} \xi^{B}+2 \phi \not D \bar{\xi}_{A}, \\
& \delta \bar{\lambda}_{A}=\frac{1}{2} \bar{\sigma}^{\mu \nu} F_{\mu \nu} \bar{\xi}_{A}+2 \not D \bar{\phi} \bar{\xi}_{A}+D_{A B} \bar{\xi}^{B}+2 \bar{\phi} \not D \xi_{A} .
\end{aligned}
$$

We can complete (2.27) to a supersymmetric configuration by introducing additional bosonic backgrounds

$$
D_{12}=-\alpha \frac{i}{\ell^{2}}\left(1-g_{\epsilon}(\theta)+\cot \theta g_{\epsilon}^{\prime}(\theta)\right), \quad \phi=\alpha \frac{g_{\epsilon}(\theta)-1}{2 \ell}, \quad \bar{\phi}=\alpha \frac{g_{\epsilon}(\theta)-1}{2 \ell} .
$$

In this background, the gaugino variations vanish with an arbitrary spinor from (2.20). In the limit $\epsilon \rightarrow 0$ we get

$$
\begin{aligned}
g_{\epsilon}(\theta) & \rightarrow 1, & g_{\epsilon}^{\prime}(\theta) & \rightarrow \delta(\theta), \\
A_{\text {defect }} & =\alpha d \tau, & F_{\text {defect }} & =\alpha \delta(\theta) d \theta \wedge d \tau, \\
D_{12}^{\text {defect }} & =-\alpha \frac{i}{\ell^{2}} \frac{\delta(\theta)}{\theta}, & \phi & =\bar{\phi}=0 .
\end{aligned}
$$

Note that both the smooth and singular configurations are off the original contour of integration for the vector multiplet (2.22) ( $D_{12}$ is imaginary).

\subsubsection{The supersymmetric Lagrange multiplier}

Following the logic of the $\mathbb{Z}_{n}$ gauge theory, we will impose the configuration for the background vector multiplet using a supersymmetric Lagrange multiplier. We make the vector multiplet dynamical and introduce an $\mathcal{N}=2$ abelian tensor multiplet $E$

$$
\left\{E_{\mu \nu}, G, \bar{G}, L^{A B}, \text { fermions }\right\},
$$

whose field strength sits in the linear multiplet $G$ [30]. ${ }^{11}$ We couple $E$ to the vector multiplet (actually its field strength) using a supersymmetric BF term [30]

$$
S_{\mathrm{BF}}^{(4 d)}=\frac{i n}{2 \pi} \int \sqrt{g}\left(\phi G+\bar{\phi} \bar{G}-\frac{1}{2} D^{A B} L_{A B}+\frac{1}{4} \varepsilon^{\mu \nu \rho \sigma} E_{\mu \nu} F_{\rho \sigma}+\text { fermions }\right) .
$$

\footnotetext{
${ }^{10}$ We write $g_{\epsilon}^{\prime}(\theta) \equiv \partial_{\theta} g_{\epsilon}(\theta)$.

${ }^{11}$ We use the letters $E$ and $G$ to indicate both the supermultiplets and some of their components. We hope this does not cause too much confusion.
} 
We then introduce a Wilson surface operator for the tensor multiplet by adding an additional copy of (2.35), but replacing all fields in the vector multiplet with their values from (2.32) and (2.33), and with $\alpha=1$,

$$
\begin{aligned}
W_{\text {surface }}(k) & \equiv \exp \left[\frac{i k}{2 \pi} \int \sqrt{g}\left(-\frac{1}{2} D_{A B}^{\text {defect }} L^{A B}+\frac{1}{4} \varepsilon^{\mu \nu \rho \sigma} E_{\mu \nu} F_{\rho \sigma}^{\text {defect }}\right)\right], \\
& =\exp \left[i k \int_{\mathbb{S}_{\theta=0}^{2}} \sqrt{g}\left(i \ell^{2} L^{12}+\star_{2} E\right)\right] .
\end{aligned}
$$

Integrating out the tensor multiplet would result in a supersymmetric delta function setting all elements of the vector multiplet to their values for the surface defect. Instead of doing this, we first perform the localization.

\subsubsection{Localization of the tensor multiplet}

The localizing term for the abelian vector multiplet can be taken from [29]. For the tensor multiplet, we must consider the transformation of the fermions in this multiplet, denoted $\varphi^{A}, \bar{\varphi}^{A} .{ }^{12}$ The variations are

$$
\begin{aligned}
& \delta \varphi^{A}=\sigma^{\mu}\left(\partial_{\mu} L^{A B}+\frac{1}{2} \varepsilon^{A B} \varepsilon_{\mu \nu \rho \sigma} \partial^{\nu} E^{\rho \sigma}\right) \bar{\xi}_{B}-G \xi^{A}+2 L^{A B} \eta_{B}, \\
& \delta \bar{\varphi}^{A}=\bar{\sigma}^{\mu}\left(\partial_{\mu} L^{A B}+\frac{1}{2} \varepsilon^{A B} \varepsilon_{\mu \nu \rho \sigma} \partial^{\nu} E^{\rho \sigma}\right) \xi_{B}-\bar{G} \bar{\xi}^{A}+2 L^{A B} \bar{\eta}_{B} .
\end{aligned}
$$

We take the localizing term

$$
S_{\text {tensor localizing term }}=\int_{\mathbb{S}^{4}} \delta\left[(\delta \varphi)_{A}^{\dagger} \varphi^{A}+(\delta \bar{\varphi})_{A}^{\dagger} \bar{\varphi}^{A}\right] .
$$

We will use reality conditions for $G, \bar{G}, L^{A B}$ appropriate for the coupling to the vector multiplet fields (2.35)

$$
\bar{G}=-G^{\dagger}, \quad\left(L^{A B}\right)^{\dagger}=-L_{A B},
$$

which yields

$$
\left(L^{11}\right)^{\dagger}=-L^{22}, \quad \operatorname{Im}\left(L^{12}\right)=0
$$

We also define

$$
\begin{aligned}
H_{\mu} & \equiv \frac{1}{2} \varepsilon_{\mu \nu \rho \sigma} \partial^{\nu} E^{\rho \sigma}, \\
G & =G_{r}+i G_{i} .
\end{aligned}
$$

\footnotetext{
${ }^{12}$ We have decomposed each spinor $\varphi^{i}$ from [30] as a doublet $\varphi^{A}, \bar{\varphi}^{A}$. Note that the definition of the Levi-Civita symbol in [30] contains an extra factor of " $i$ " compared to the usual definition: $\varepsilon^{1234}=1$. Examination of the gravitino transformation in [30] yields the following dictionary in relation to [29]

$$
\epsilon^{i} \rightarrow\left(\begin{array}{l}
\xi^{A} \\
\bar{\xi}^{A}
\end{array}\right), \quad \eta^{i} \rightarrow \frac{1}{2} \not D\left(\begin{array}{l}
\xi^{A} \\
\bar{\xi}^{A}
\end{array}\right)=-2 i\left(\begin{array}{l}
\xi^{\prime A} \\
\bar{\xi}^{\prime A}
\end{array}\right)
$$

Superscripts on both sides indicate $\mathrm{SU}(2)_{R}$ transformations. Note the factor of 2 difference with the $\eta$ used in e.g. [31, 32].
} 
The bosonic part of the resulting localizing term is

$$
\begin{aligned}
S_{\text {tensor localizing term }}=\int_{\mathbb{S}^{4}} & {\left[2 H^{\mu} H_{\mu}+2\left|\partial_{\mu} L^{12}\right|^{2}+2\left|\left(\partial_{\mu}+\frac{i}{\ell} v_{\mu}\right) L^{11}\right|^{2}\right.} \\
& \left.+\frac{1}{\ell^{2}}\left(8-2 v^{\mu} v_{\mu}\right)\left|L^{11}\right|^{2}+2\left(G_{r}\right)^{2}+2\left(G_{i}+2 \frac{L^{12}}{\ell}\right)^{2}\right] .
\end{aligned}
$$

The vector field $v_{\mu}$ is such that

$$
\nabla^{\mu} v_{\mu}=0, \quad\left(8-2 v^{\mu} v_{\mu}\right)>0 .
$$

The localization locus is thus

$$
H_{\mu}=0, \quad L^{11}=L^{22}=G_{r}=0, \quad G_{i}=-2 \frac{L^{12}}{\ell}=G_{0} .
$$

with $G_{0}$ a real constant.

\subsubsection{Modification of the matrix model}

We will couple the vector multiplet representing the surface defect to the physical vector multiplets and hypermultiplets of the $\mathcal{N}=2$ SCFT as detailed in section 3.1. After localization, the matrix model representing the $\mathbb{S}^{4}$ partition function is modified by the presence of the defect. We would like to show that this modification amounts to giving the hypermultiplets or the vector multiplets an imaginary mass, given by $-i \frac{k}{n}$. Below we discuss all contributions of $V$ and $E$ to the matrix model.

Classical contribution. The multiplets $V$ and $E$ have a classical action given by (2.35) and (2.36). After localization, these terms give insertions in the matrix model. Using the vevs for the scalar fields

$$
\phi=\bar{\phi}=-i \frac{\sigma}{2}, \quad D_{12}=\frac{\sigma}{\ell},
$$

and

$$
G=\bar{G}=i G_{0}, \quad L^{12}=-\frac{\ell}{2} G_{0},
$$

we get

$$
\begin{aligned}
\exp \left(-S_{\mathrm{BF}}^{(4 d)}\right) & \rightarrow \exp \left(-2 \pi i \ell^{4} n \sigma G_{0}\right) \\
W_{\text {surface }}(k) & \rightarrow \exp \left(2 \pi \ell^{3} k G_{0}\right)
\end{aligned}
$$

Perturbative contributions. The quadratic approximation to the localizing term (2.44) for the tensor multiplet around the locus (2.46) is independent of $G_{0}$. The one-loop determinant is thus a $G_{0}$ independent number, which is furthermore equal to the partition function of a free tensor multiplet on the round four-sphere. This multiplet can be dualized into an uncharged massless hypermultiplet whose partition function can be deduced from the expressions in 3.5. The one-loop determinant for $A$ is trivial. 
Non-perturbative contributions. In the absence of two-cycles, the equation

$$
H=\star d E=0,
$$

implies that we can set

$$
E=0
$$

up to tensor gauge transformations. When there are non-trivial two-cycles, the form $E$ is closed and the tensor gauge transformations imply that $E$ is gauge equivalent to 0 whenever it represents an integral class. The remaining moduli of $E$ can be identified with the values of the possible Wilson surfaces. The gauge field $A$ also has moduli in this situation. A smooth instanton configuration for $A$ may exist, where $A$ is a non-trivial connection with integral flux on the two-cycle.

The analysis above applies only to smooth configurations for $A, E$ and their gauge transformation parameters. Experience shows that we should allow singular configurations for $A$, as we did for the non-abelian gauge fields. The coupling of $E$ to the field strength $F_{A}$ means that we should consider allowing singular configurations for $E$ as well, at least at the poles. In fact, the configuration (2.32) which we are trying to reproduce has a singularity on the entire maximal two-sphere. If we allow $E$ to have the singularities at the same position as $A$, then the coupling

$$
\frac{i n}{2 \pi} \int F_{A} \wedge E \quad \subset \quad \frac{i n}{2 \pi} \int V G
$$

induces a non-vanishing classical contribution upon localization

$$
\frac{i n}{2 \pi} \int F_{A} \wedge E \rightarrow \frac{i n}{2 \pi} \int F_{A}^{\text {inst }} \wedge E^{\text {classical }} .
$$

The form $E$ is again closed and the tensor gauge transformations again imply that $E$ is gauge equivalent to 0 whenever it represents an integral class. The remaining moduli of $E$, parameterizing singular closed two-forms localized at the poles modulo integral forms, are angular parameters. Upon localization, there is a contribution to the matrix model of the form

$$
n \int F_{A} \wedge E \rightarrow n \int F_{A}^{\text {inst }} \wedge[\alpha]
$$

where $F_{A}^{\text {inst }}$ is the field strength of some singular instanton configuration at the poles and $[\alpha]$ is a representative of any singular two-form at the poles, which is defined only up to integral classes. The part of the electric defect containing an integral over $E$ is, however, trivial

$$
\oint_{\partial \Sigma} E=\oint_{\Sigma} H=0
$$

because $E$ is being paired with a trivial cycle represented by $\partial \Sigma$. Integration of

$$
\exp \left(\operatorname{in} \int F_{A}^{\mathrm{inst}} \wedge[\alpha]\right)
$$

over all $\alpha$ then restricts $F_{A}^{\text {inst }}$ to vanish. This means that we only need to work in the instanton number 0 sector for $A$. 
Integrating out. In addition to the above, there is a remaining integration over $G_{0}$. This integral, and the one over $\sigma$, can be done explicitly. The result is simply to set

$$
\sigma \rightarrow-\frac{i}{\ell} \frac{k}{n}
$$

or in the units of the rest of the sections

$$
\sigma=-i \frac{k}{n}
$$

This was what we set out to show.

The parameter entering the one-loop and instanton contributions in the matrix model, described in section 3, is $i m$, where $m$ is identified with the $\sigma$ of a background vector multiplet. Hence, our surface operator is equivalent to an imaginary mass

$$
m=-i \frac{k}{n},
$$

equivalently, a shift of the parameters by

$$
q_{\text {surface }}=\frac{k}{n} .
$$

\subsection{The $5 d \mathcal{N}=1$ codimension-two defect}

We describe the implementation of codimension-two defect operators in five-dimensional $\mathcal{N}=1$ theories. Most of the analysis is similar to the four-dimensional case, so we will be brief. Conventions and notations are the same as in [14].

\subsubsection{Killing spinors on the five-sphere}

Supersymmetric field theories on curved spaces are systematically obtained in the rigid limit of the $\mathcal{N}=1$ supergravity in five dimensions which has an $\mathrm{SU}(2)_{R}$ symmetry whose indices are denoted by $A, B$ as in the $4 d$ case. There are $\mathrm{SU}(2)_{R}$ gauge field $V_{\mu}^{A B}, \mathrm{SU}(2)_{R}$ triplet scalar field $t^{A B}$ and the other fields which are irrelevant to the following discussion in the Weyl multiplet [33]. The variations of the fermions in the multiplet have to vanish for preserving supersymmetries on given background fields and the solutions are the Killing spinors. We set the radius of the five-sphere to one from the beginning to simplify the discussion.

The round unit five-sphere allows the Killing spinor in the coordinates

$$
d s_{\mathbb{S}^{5}}^{2}=d \theta^{2}+\sin ^{2} \theta d \tau^{2}+\cos ^{2} \theta d s_{\mathbb{S}^{3}}^{2}
$$

when the background fields are set to $[14,34]$

$$
t_{B}^{A}=\frac{1}{2}\left(\sigma_{3}\right)_{B}^{A}, \quad V_{B}^{A}=-\frac{i}{2}\left(\sigma_{3}\right)_{B}^{A} d \tau, \quad \text { others }=0 .
$$

With the hermitian gamma matrices in tensor product forms

$$
\Gamma^{1}=\sigma^{1} \otimes \mathbf{1}_{2}, \quad \Gamma^{2}=\sigma^{2} \otimes \mathbf{1}_{2}, \quad \Gamma^{i+2}=\sigma^{3} \otimes \sigma^{i}, \quad(i=1,2,3),
$$


a spinor $\xi^{A}$ in five dimensions is also written as tensor products of spinors $\xi^{A}$ and $\eta^{A}$ in two and three dimensions

$$
\xi^{A}=\xi^{A} \otimes \eta^{A}
$$

The Killing spinor on the round sphere (2.61) is given by

$$
\begin{array}{ll}
\xi^{1}=\left(e^{\frac{i}{2} \theta \sigma_{1}} \zeta^{1}\right) \otimes \eta_{+}, & \sigma_{3} \zeta^{1}=\zeta^{1}, \\
\xi^{2}=\left(e^{-\frac{i}{2} \theta \sigma_{1}} \zeta^{2}\right) \otimes \eta_{-}, & \sigma_{3} \zeta^{2}=-\zeta^{2}
\end{array}
$$

where $\zeta^{1,2}$ are constant spinors and $\eta_{ \pm}$are the Killing spinors on a unit three-sphere

$$
\left(\partial_{i}+\frac{i}{2} \sigma_{i}\right) \eta_{ \pm}= \pm \frac{i}{2} \sigma_{i} \eta_{ \pm}
$$

\subsubsection{Codimension-two defects}

A surface defect in a flavor symmetry is specified as a singular configuration in a background $\mathcal{N}=1$ abelian vector multiplet

$$
\left\{A_{\mu}, \sigma, Y^{A B}, \lambda^{A}\right\}
$$

where $A_{\mu}$ the gauge field, $\sigma$ a real scalar, $Y^{A B}$ an $\mathrm{SU}(2)_{R}$ triplet scalar and $\lambda^{A}$ an $\mathrm{SU}(2)_{R^{-}}$ Majorana fermion, respectively.

We want a codimension-two surface defect at $\theta=0$ on the round sphere. Introducing a smoothing function $g_{\epsilon}(\theta)$ as in the four-dimensional case (2.28), the background gauge field describing the defect is

$$
A_{\text {defect }}=\alpha g_{\epsilon}(\theta) d \tau,
$$

whose field strength becomes

$$
F_{\text {defect }}=\alpha g_{\epsilon}^{\prime}(\theta) d \theta \wedge d \tau \text {. }
$$

This configuration is supersymmetric if the real scalar $\sigma_{\text {defect }}$, the gaugino $\lambda_{\text {defect }}^{A}$ and the triplet scalar $Y_{\text {defect }}^{A B}$ take the following forms

$$
\left(Y_{\text {defect }}\right)_{B}^{A}=-\frac{i}{2} \alpha g_{\epsilon}^{\prime}(\theta)\left(\sigma_{3}\right)_{B}^{A}, \quad \sigma_{\text {defect }}=\lambda_{\text {defect }}^{A}=0
$$

This configuration is invariant under the supersymmetric transformation, especially one sees

$$
0=\delta \lambda_{\text {defect }}^{A}=\frac{1}{4} \Gamma^{\mu \nu} \xi^{A}\left(F_{\text {defect }}\right)_{\mu \nu}+\left(Y_{\text {defect }}\right)_{B}^{A} \xi^{B} .
$$

One can replace $g_{\epsilon}^{\prime}(\theta)$ with the delta function $\delta(\theta)$ in the singular limit $\epsilon \rightarrow 0$. 


\subsubsection{Defects and supersymmetric Lagrange multiplier}

We will implement defects in a flavor symmetry by coupling the abelian vector multiplet to the linear multiplet

$$
\left\{L^{A B}, E_{\mu \nu}, N, \varphi^{A}\right\}
$$

consisting of an $\mathrm{SU}(2)_{R}$ triplet scalar $L^{A B}$, an antisymmetric tensor gauge field $E_{\mu \nu}$, a real scalar $N$ and an $\mathrm{SU}(2)_{R}$-Majorana fermion $\varphi^{A}$, through a supersymmetric BF coupling [33]

$$
S_{\mathrm{BF}}^{(5 d)}=\frac{i n}{\pi} \int \sqrt{g}\left(\frac{1}{4} F_{\mu \nu} E^{\mu \nu}+Y_{A B} L^{A B}-\frac{1}{2} \sigma N+2 i \lambda \varphi\right) .
$$

It is gauge invariant for an integer $n$ as $E_{\mu \nu}$ transforms as $\delta_{\text {gauge }} E^{\mu \nu}=\partial_{\rho} \Lambda^{\mu \nu \rho}$. The supersymmetric transformation laws of the linear multiplet with only the backgrounds $t^{A B}$ and $V^{A B}$ turned on are given by

$$
\begin{aligned}
\delta L^{A B} & =2 i \xi^{(A} \varphi^{B)}, \\
\delta E^{\mu \nu} & =2 i \xi \Gamma^{\mu \nu} \varphi, \\
\delta N & =-2 \xi \Gamma^{\mu}\left(D_{\mu} \varphi\right)-10 i \xi^{A} \varphi^{B} t_{A B}, \\
\delta \varphi^{A} & =i\left(D_{\mu} L^{A B}\right) \Gamma^{\mu} \xi_{B}-4 t_{C}^{B} L_{B}^{C} \xi^{A}-6 t_{C}^{(A} L^{B) C} \xi_{B}+\frac{i}{2} \Gamma_{\mu} \xi^{A} \partial_{\nu} E^{\mu \nu}+\frac{1}{2} \xi^{A} N .
\end{aligned}
$$

In addition, we introduce a surface operator for the linear multiplet with the BF coupling to the defect abelian vector multiplet

$$
\begin{aligned}
W_{\text {surface }}(k) & =\exp \left[-\frac{i k}{2 \pi} \int \sqrt{g}\left(-\left(Y^{\text {defect }}\right)_{A}^{B} L_{B}^{A}+\frac{1}{4} F_{\mu \nu}^{\text {defect }} E^{\mu \nu}\right)\right], \\
& =\exp \left[-i k \alpha \int_{\mathbb{S}_{\theta=0}^{3}}\left(-\frac{i}{2}\left(L^{1}{ }_{1}-L_{2}^{2}\right) \omega_{3}+\frac{1}{2} * E\right)\right],
\end{aligned}
$$

where $\omega_{3}$ is the volume form of the unit three-sphere and $*$ is the Hodge operator in five dimensions. Choosing $\alpha=2$ the defect operator is gauge invariant for an integer $k$.

The linear multiplet plays a role of the Lagrange multiplier and integrating it out in the path integral with the BF term and the Wilson surface results in setting the vector multiplet to the defect configuration.

Instead of doing so, we localize the tensor multiplet on the round sphere with the localizing term

$$
V_{\text {tensor }}^{(\text {loc }}=\delta\left[\left(-i\left(D_{\mu} L_{B}^{A}\right) \xi^{B} \Gamma^{\mu}-4 t_{C}^{B} L_{B}^{C} \xi^{A}-6 t_{C}^{(A} L^{B) C} \xi_{B}-\frac{i}{2}\left(\partial_{\nu} E^{\mu \nu}\right) \xi^{A} \Gamma_{\mu}+\frac{1}{2} N \xi^{A}\right) \varphi_{A}\right],
$$

whose bosonic part is written as

$$
\frac{1}{2}\left(D_{\mu} L_{B}^{A}\right)\left(D^{\mu} L_{A}^{B}\right)+\frac{1}{4}\left(\partial^{\nu} E_{\mu \nu}\right)\left(\partial_{\nu} E^{\mu \nu}\right)+\frac{1}{4}\left(N-8 \hat{L}_{A}^{A}\right)^{2}+18 \hat{L}^{(A B)} \hat{L}_{(A B)},
$$

where we defined $\hat{L}^{A B} \equiv t_{C}^{A} L^{C B}$. 
In localizing the tensor multiplet, we impose the reality condition for $L_{B}^{A}$

$$
\left(L_{B}^{A}\right)^{\dagger}=L_{A}^{B}
$$

that is equivalent to

$$
\left(\hat{L}_{A}^{A}\right)^{\dagger}=\hat{L}_{A}^{A}, \quad\left(\hat{L}^{(A B)}\right)^{\dagger}=L_{(A B)} .
$$

This choice makes the bosonic part of the localizing term be semi-positive definite and the tensor multiplet localizes to

$$
D_{\mu} L_{B}^{A}=0, \quad \partial_{\nu} E^{\mu \nu}=0, \quad N=8 \hat{L}_{A}^{A}, \quad \hat{L}_{(A B)}=0,
$$

which on the round sphere yields

$$
N=4\left(L_{1}^{1}-L_{2}^{2}\right) \equiv N_{0}=\text { const }, \quad L_{2}^{1}=L_{1}^{2}=0 .
$$

Localizing the vector multiplet to the fixed locus

$$
A_{\mu}=0, \quad Y_{A B}=0, \quad \sigma=\text { const },
$$

the BF term (2.73) and the surface operator (2.75) end up with

$$
\begin{aligned}
\exp \left(-S_{\mathrm{BF}}^{(5 d)}\right) & \rightarrow \exp \left(\frac{i \pi^{2}}{2} n \sigma N_{0}\right) \\
W_{\text {surface }}(k) & \rightarrow \exp \left(-\frac{\pi^{2}}{2} k N_{0}\right) .
\end{aligned}
$$

the integration over $N_{0}$ sets

$$
\sigma=-i \frac{k}{n}
$$

This value is consistent with the mass shift by the parameter (2.60) as in the $4 d$ case as we will confirm in section 3 .

\section{Squashing from defects}

We are now in a position to demonstrate the relationship between the supersymmetric Rényi entropy and supersymmetric codimension-two defects. In the original setup, the supersymmetric Rényi entropy is defined as

$$
S_{n}^{\text {susy }} \equiv \frac{1}{1-n} \log \left|\frac{Z_{n}^{\text {susy }}}{\left(Z_{1}\right)^{n}}\right|
$$

where $Z_{n}$ is the appropriate supersymmetry preserving partition function on the branched $d$-sphere, or the squashed $d$-sphere. The schematic form of the localization calculation for $Z_{n}$ is

$$
Z_{n}=\sum \int_{\text {moduli }}\left[Z_{n}^{\text {classical }}(\text { moduli }) Z_{n}^{\text {pert }}(\operatorname{moduli})\right]
$$


where the sum/integral is over the moduli space of supersymmetric zero modes, $Z_{n}^{\text {classical }}$ is the exponential of minus the Euclidean action evaluated on the moduli space, and $Z_{n}^{\text {pert }}$ is the one-loop determinant obtained by evaluating the path integral over non-zero modes in the quadratic approximation around the moduli space.

The partition function for the $n$-copy theory on the round sphere is simply

$$
\left(Z_{1}\right)^{n}
$$

We would like to demonstrate that $Z_{n}$ can be computed using $Z_{1}$ and the insertion of defects, i.e.

$$
Z_{n}=Z_{n}^{\text {defect }}
$$

Where the right hand side is the partition function of the $n$-copy theory on the round sphere in the presence of a specific codimension-two defect described in the next subsection. After the linear field redefinition, the result is

$$
Z_{n}^{\text {defect }} \equiv \sum \int_{n \text { moduli }}\left[\delta_{\text {moduli }} \prod_{k=0}^{n-1} Z_{1}^{\text {classical }}(\text { moduli }) Z_{1}^{\text {pert-defect }}(k, \text { moduli })\right]
$$

The outer sum/integral is over $n$ copies of the moduli. The symbol $\delta_{\text {moduli }}$ is a placeholder for the sewing operation which identifies how the squashed sphere moduli space fractionalizes into $n$ copies. $Z_{1}^{\text {classical }}$ is the classical contribution of a single copy of the theory evaluated on the round sphere. $Z_{1}^{\text {pert-defect }}(k$, moduli $)$ is the perturbative contribution, in the quadratic approximation around the moduli space, in the presence of the appropriate defect for the $k^{\text {th }}$ copy.

\subsection{Coupling defects to the $n$-copy theory}

The superfield $V$, which carries the information about the supersymmetric defect, must be coupled to the physical fields of the $n$-copy theory. For matter multiplets, this is the usual minimal coupling of $V$ to chiral multiplets or hypermultiplets. We need not consider the effect of the defect on the other terms in the action involving matter fields, since these vanish at the level of the quadratic approximation around the localization locus for any of the setups we consider. The coupling of a flat connection carried by $V$ to physical gauge fields can be accomplished by formally performing the field redefinition in the introduction. Since non-abelian gauge fields are not free fields at finite gauge coupling, the resulting action would inevitably look like a gauge non-invariant mess. However, gauge invariance, with the caveats already mentioned, is guaranteed by the ability to undo the field redefinition.

If the gauge group is $\mathrm{SU}(N)$, there is a physical procedure which implements the right defect and makes clear the form of the coupling to a background vector, at the level of the quadratic approximation to the moduli space. We use the language of $4 d \mathcal{N}=2$, but the same applies to any of the theories under consideration. 
First, consider the enlarged gauge group $\mathrm{U}(n N)$, where the gauge group of the $n$-copy theory, $\mathrm{SU}(N)^{n}$, is embedded as a block diagonal subgroup

$$
\left(\begin{array}{cccc}
\mathrm{SU}(N)_{0} & 0 & \cdots & 0 \\
0 & \mathrm{SU}(N)_{1} & \cdots & 0 \\
\vdots & \vdots & \ddots & \vdots \\
0 & 0 & \cdots & \mathrm{SU}(N)_{n-1}
\end{array}\right)
$$

The required codimension-two defect, before the field redefinition, can be viewed as a flat connection, on $\mathbb{S}^{d} \backslash \partial \Sigma$, which is represented by a one-form with holonomy

$$
\left(\begin{array}{ccccc}
0 & 1 & 0 & \cdots & 0 \\
0 & 0 & 1 & \cdots & 0 \\
\vdots & \vdots & & \ddots & \vdots \\
0 & 0 & 0 & \cdots & 1 \\
1 & 0 & 0 & \cdots & 0
\end{array}\right)
$$

along any path encircling $\partial \Sigma$ once in a chosen direction. To preserve supersymmetry, we consider a Gukov-Witten type surface defect with the holonomy (3.7) specifying the data $[35,36]$.

To go back to the $n$-copy theory, while keeping the defect, one should first Higgs $\mathrm{U}(n N)$ down to $\mathrm{SU}(N)^{n}$ by giving an appropriate large vev to the adjoint scalar in the vector multiplet

$$
X^{\text {Higgs }}=\left(\begin{array}{cccc}
t_{0} & 0 & \cdots & 0 \\
0 & t_{1} & \cdots & 0 \\
\vdots & \vdots & \ddots & \vdots \\
0 & 0 & \cdots & t_{n-1}
\end{array}\right) .
$$

Upon taking $t_{i} \rightarrow \infty$, all modes not coming from the original $n$-copy theory are infinitely massive and do not contribute to the computation. $X^{\text {Higgs }}$ should really be considered only up to permutations of the $t_{i}$, which are a part of the Weyl group of the theory. In fact, the holonomy (3.7) acting on $X^{\text {Higgs }}$ produces such a permutation. Although we do not show this explicitly, we take this to mean that the Gukov-Witten operator with this data preserves the same supersymmetry as $X^{\text {Higgs }}$. After a change of variables, which is in this case a constant $\mathrm{SU}(n N)$ gauge transformation, the fields in the vector multiplet for $\mathrm{SU}(N)_{k}$ acquire a monodromy $\exp (2 \pi i k / n)$ around $\partial \Sigma .^{13}$

The physical effect of the defect on vector multiplets can now be examined more carefully at the level of the quadratic approximation to the moduli space of the remaining light fields. Since the action in this approximation is quadratic, the monodromy can be traded for a coupling to a background vector multiplet with a specific profile. This profile is singular, and determined by the value of the monodromy and by supersymmetry. It is this multiplet, denoted by $V$, which arises in our realization of the supersymmetric $\mathbb{Z}_{n}$ gauge theory.

\footnotetext{
${ }^{13}$ Note that the diagonal elements of $\mathrm{SU}(N)_{k}$ also acquire this monodromy. Had we tried to implement the defect using a Gukov-Witten type surface defect in each $\mathrm{SU}(N)_{k}$, this would not have been so.
} 


\subsection{Mass terms and vortices}

A vev for the scalar components of $V$ appears as a supersymmetric mass term. For vector multiplets, this is a mass term of the Higgs type. For matter multiplets, it is a mass term associated with the U(1) flavor symmetry of a free hypermultiplet or chiral multiplet. As shown in section 2, the vortex charge is equivalent, in the matrix model, to an imaginary mass term. Any required vortex charge can be produced using the supersymmetric codimension-two defects of section $2 .{ }^{14}$ The supersymmetric $\mathbb{Z}_{n}$ gauge theory naturally produces vortex charges of the type $k / n$. One still needs to show that taking these vortex charges reproduces the partition function on the squashed sphere. We show this for each element of the matrix model in the following subsections.

To reproduce the squashed sphere result using defects, one must correctly choose the origin of the imaginary part of the mass deformation parameters in both the perturbative and non-perturbative parts. As shown in [37], there is a subtlety in doing so for the hypermultiplet mass of the $4 d \mathcal{N}=2^{*}$ theory. Only by choosing this origin correctly can one produce a Gaussian matrix model for Wilson loops in $\mathcal{N}=4$, which was the topic of [28]. The correct origin for the mass deformation is associated with enhanced supersymmetry, and with the vanishing of the instanton contributions for $\mathcal{N}=4$. As shown in [37], for the $\mathcal{N}=2^{*}$ theory, the mass parameter usually used in the Nekrasov partition function should be shifted as ${ }^{15}$

$$
m_{f} \rightarrow m_{f}+\frac{1}{2}\left(\epsilon_{1}+\epsilon_{2}\right)
$$

in order to align it with the mass parameter used in the perturbative part. This shift comes from careful examination of the equivariant action of the square of the supercharge used in the localization, and so it should apply to any $\mathcal{N}=2$ theory. In terms of the squashing parameters $\omega_{1,2}$ it is

$$
m_{f} \rightarrow m_{f}+\frac{1}{2}\left(\omega_{1}+\omega_{2}\right),
$$

which applies equally well at the north and south poles. The analogous statement in the $5 d \mathcal{N}=1^{*}$ theory is

$$
m_{f} \rightarrow m_{f}+\frac{1}{2}\left(\omega_{1}+\omega_{2}+\omega_{3}\right) .
$$

In addition to aligning the mass parameters, one must determine the value of $m_{f}$ at the conformal fixed point. According to [37], the round four-sphere $\left(\epsilon_{1}=\epsilon_{2}\right)$ value, after the shift, is

$$
m_{f}=0 .
$$

At this value, one recovers a Gaussian matrix model for $\mathcal{N}=2^{*}$, which is interpreted as a signal of supersymmetry enhancement to $\mathcal{N}=4$. On the squashed sphere, one must take [38]

$$
m_{f}= \pm \frac{1}{2}\left(\epsilon_{1}-\epsilon_{2}\right),
$$

\footnotetext{
${ }^{14}$ We have shown that this is so for any fraction $\frac{k}{n}$. By continuity, the same is true for any real number.

${ }^{15}$ In [37], a mass deformation parameter $m$ appears. It should be identified with $m_{f}$ in our notation.
} 
to achieve the same effect. In terms of the squashing parameters, this is

$$
m_{f}= \pm \frac{1}{2}\left(\omega_{1}-\omega_{2}\right) \text {. }
$$

We will choose the upper sign.

For the $5 d \mathcal{N}=1^{*}$ model, the prescription described in $[39,40]$ is to shift the mass term by an additional amount

$$
\pm \frac{1}{2}\left(\omega_{1}+\omega_{2}-\omega_{3}\right)
$$

This choice was made so as to make contact with the $4 d$ results in [37]. We will, instead, use an additional shift by

$$
-\frac{1}{2}\left(\omega_{2}+\omega_{3}-\omega_{1}\right)
$$

which has the property that it reproduces the $4 d$ result for the instanton partition function after taking $\beta \rightarrow 0$.

Combining the shifts gives the following mass parameter for the instanton partition function

- For the $4 d$ calculation $m_{f}=\omega_{1}$, which corresponds to $m_{f}=\epsilon_{1}, \epsilon_{2}$ at the north and south pole respectively.

- For the $5 d$ calculation $m_{f}=\omega_{1}$, which corresponds to $m_{f}=\epsilon_{1}, \epsilon_{2}, \frac{2 \pi}{\beta}$ at the three fixed points.

We will denote these values, in any dimension, as $m_{f}^{0}$. Note that the one-loop part does not require the first shift, so its effective mass parameter is different.

A codimension-two defect operator, with charge $\frac{k}{n}$, further shifts the mass parameter by

$$
m_{f} \rightarrow m_{f}+\frac{k}{n}
$$

We will show that such shifts, which can be interpreted in terms of the $\mathbb{Z}_{n}$ gauge theory, are enough to reproduce the supersymmetric Rényi entropy in four and five dimensions. Had we not implemented the second shift above, a different vortex charge would have been required for the hypermultiplets. This additional vortex charge can be thought of as an additional twist arising from the R-charge of a hypermultiplet. It can be incorporated by adjusting the holonomy (3.7) to include an overall phase associated with $\mathrm{U}(1) \subset \mathrm{U}(n N)$. The result in three dimensions does not include instantons and the vortex charge is

$$
q_{k}^{3 d \text { vortex }}=\frac{\Delta}{2}\left(\frac{1}{n}-1\right)+\frac{k}{n},
$$

where $\Delta$ is the R-charge of the chiral multiplet. A vector multiplet can be interpreted as a chiral multiplet with $\Delta=0$. The addition of a term proportional to $\Delta$ was interpreted in [6] as an additional R-symmetry twist of the usual Rényi entropy, which is needed to make it supersymmetric. It should be noted that, if all chiral multipets have the same $\Delta$, this term too can be canceled by shifting the origin of the mass term for chiral multiplets in the imaginary direction, as explained above. The result is a supersymmetric observable, with or without this additional shift. We do not currently know of a criterion which makes one of these options more relevant. 


\subsection{The matrix models}

We collect the expressions for the matrix models associated to the squashed sphere partition functions in three, four and five dimensions. Our conventions for integration over the Lie algebra are in appendix A. Special functions in the one-loop determinants and instanton contributions are defined in appendices $\mathrm{B}$ and $\mathrm{C}$, respectively.

The matrix model for a $3 d \mathcal{N}=2$ theory on the squashed sphere is [41, 42]

$$
\begin{array}{r}
Z^{\text {susy } 3 d}=\frac{1}{|W|} \int \prod_{i=1}^{\mathrm{rank} G} \frac{d \sigma_{i}}{\sqrt{\omega_{1} \omega_{2}}} e^{\frac{\pi i \kappa}{\omega_{1} \omega_{2}} \operatorname{Tr}\left(\sigma^{2}\right)} \cdot \prod_{\alpha \in \Delta_{+}} S_{2}(i \alpha(\sigma) \mid \boldsymbol{\omega}) S_{2}(-i \alpha(\sigma) \mid \boldsymbol{\omega}) \\
\cdot \prod_{I=1}^{\# \text { of chirals }} \prod_{\rho \in \mathcal{R}_{I}} S_{2}\left(i \rho(\sigma)+i m_{I}+\frac{|\boldsymbol{\omega}|}{2} \Delta_{I} \mid \boldsymbol{\omega}\right)^{-1},
\end{array}
$$

- $\sigma_{i}$ runs over the Cartan of the Lie algebra $\mathfrak{g}$ of the group $G$, which we have assumed is $\mathrm{U}(N)$. Our conventions are such that $\sigma_{i}$ are real (see appendix A).

- $\Delta_{+}$are the positive roots of $\mathfrak{g}$. $\rho$ denotes a weight in the representation $\mathcal{R}_{I}$ associated to the $I^{\text {th }}$ chiral multiplet.

- $|W|$ is the size of the Weyl group.

- $\Delta_{I}$ is the R-charge of the $I^{\text {th }}$ chiral multiplet.

- $\kappa$ is the Chern-Simons level.

- $\boldsymbol{\omega}=\left(\omega_{1}, \omega_{2}\right)$ are squashing parameters, and

$$
|\boldsymbol{\omega}| \equiv\left|\sum \omega_{i}\right|=\left|\omega_{1}+\omega_{2}\right|
$$

The supersymmetric Rényi entropy is computed using $\boldsymbol{\omega}=\left(1, \frac{1}{n}\right)$.

- We will set the mass parameters $m_{I}$ to zero.

The matrix model for a $4 d \mathcal{N}=2$ theory on the squashed sphere is [29]

$$
\begin{aligned}
Z^{\text {susy } 4 d}= & \frac{1}{|W|} \int \prod_{i=1}^{\mathrm{rank} G} \frac{d \sigma_{i}}{\sqrt{\omega_{1} \omega_{2}}} e^{-\frac{8 \pi^{2}}{\omega_{1} \omega_{2} g_{Y M}^{2}} \operatorname{Tr}\left(\sigma^{2}\right)} \cdot\left|Z_{\text {inst }}^{(4 d)}\left(\mathfrak{q}^{(4 d)}, i \sigma, m_{f}^{0}, \omega_{1}, \omega_{2}\right)\right|^{2} \\
& \cdot \prod_{\alpha \in \Delta_{+}} \Upsilon(i \alpha(\sigma) \mid \boldsymbol{\omega}) \Upsilon(-i \alpha(\sigma) \mid \boldsymbol{\omega}) \cdot \prod_{\rho \in \mathcal{R}} \Upsilon\left(i \rho(\sigma)+i m+\frac{|\boldsymbol{\omega}|}{2} \mid \boldsymbol{\omega}\right)^{-1}
\end{aligned}
$$

- $g_{\mathrm{YM}}$ is the Yang-Mills coupling. $g_{\mathrm{YM}}$ and $\theta_{\mathrm{YM}}$ appear also in the instanton part of the matrix model. $\mathfrak{q}^{(4 d)}$ is defined in appendix $\mathrm{C}$.

- $\mathcal{R}$ is now the total representation of the hypermultiplets.

- We have set all hypermultiplet masses to a common value: $m$. 
- The supersymmetric Rényi entropy is computed using $\boldsymbol{\omega}=\left(1, \frac{1}{n}\right)$, with the mass parameter $m$ for all hypermultiplets set to a common value

$$
m=-i\left(m_{f}^{0}-\frac{|\boldsymbol{\omega}|}{2}\right) .
$$

The result for a $5 d \mathcal{N}=1$ theory on the squashed sphere is still conjectural [34, 43-49]. We follow the form in [50]

$$
\begin{aligned}
Z^{\text {susy } 5 d} & =\frac{1}{|W|} \int \prod_{i=1}^{\operatorname{rank} G}\left(S_{3}^{\prime}(0 \mid \boldsymbol{\omega}) \frac{d \sigma_{i}}{2 \pi}\right) e^{\frac{1}{\omega_{1} \omega_{2} \omega_{3}}\left[-\frac{8 \pi^{3}}{g_{\mathrm{YM}}^{2}} \operatorname{Tr} \sigma^{2}+i \frac{\pi}{3} \kappa \operatorname{Tr} \sigma^{3}\right]} \\
& \cdot \prod_{\alpha \in \Delta_{+}} S_{3}(i \alpha(\sigma) \mid \boldsymbol{\omega}) S_{3}(-i \alpha(\sigma) \mid \boldsymbol{\omega}) \prod_{\rho \in \mathcal{R}} S_{3}\left(i \rho(\sigma)+i m+\frac{|\boldsymbol{\omega}|}{2} \mid \boldsymbol{\omega}\right)^{-1} \\
& \cdot Z_{\text {inst }}\left(\mathfrak{q}, i \sigma, m_{f}^{0}, \omega_{1}, \omega_{2}, \frac{2 \pi}{\omega_{3}}\right) Z_{\text {inst }}\left(\mathfrak{q}, i \sigma, m_{f}^{0}, \omega_{3}, \omega_{1}, \frac{2 \pi}{\omega_{2}}\right) Z_{\text {inst }}\left(\mathfrak{q}, i \sigma, m_{f}^{0}, \omega_{2}, \omega_{3}, \frac{2 \pi}{\omega_{1}}\right),
\end{aligned}
$$

- $\kappa$ is the Chern-Simons coupling. $\kappa$ and $g_{\mathrm{YM}}$ appear also in the instanton part. $\mathfrak{q}$ is defined in appendix C.

- The derivative of the triple sine function in the integral measure can be written into [51]

$$
S_{3}^{\prime}(0 \mid \boldsymbol{\omega})=\frac{\rho_{3}\left(\omega_{1}, \omega_{2}, \omega_{3}\right)^{2} \rho_{1}\left(\omega_{1}\right) \rho_{1}\left(\omega_{2}\right) \rho_{1}\left(\omega_{3}\right)}{\rho_{2}\left(\omega_{1}, \omega_{2}\right) \rho_{2}\left(\omega_{2}, \omega_{3}\right) \rho_{2}\left(\omega_{3}, \omega_{1}\right)}
$$

where $\rho_{r}(\boldsymbol{\omega})$ is the Stirling modular form

$$
\rho_{r}(\boldsymbol{\omega}):=\lim _{z \rightarrow 0} \frac{1}{z \Gamma_{r}(z \mid \boldsymbol{\omega})} .
$$

Note that $\rho_{1}(\omega)=\sqrt{2 \pi / \omega}$.

- $\boldsymbol{\omega}=\left(\omega_{1}, \omega_{2}, \omega_{3}\right)$ are squashing parameters, and $|\boldsymbol{\omega}|=\left|\omega_{1}+\omega_{2}+\omega_{3}\right|$. The supersymmetric Rényi entropy is computed using $\boldsymbol{\omega}=\left(1,1, \frac{1}{n}\right)$, and setting

$$
m=-i\left(m_{f}^{0}-\frac{|\boldsymbol{\omega}|}{2}\right) \text {. }
$$

\subsection{The scalar moduli space and classical contributions}

All of the theories we consider have a moduli space which is partially given by the vev of a Lie algebra valued scalar. The scalar $\sigma$ has eigenvalues $\vec{\sigma}$. Integration over $\sigma$ is what makes the result of the localization procedure into a matrix model. In our setup for the $n$-copy theory, there is one such $\sigma_{k}$ and one integration for each copy. There are also classical contributions to the matrix model which depend on $\sigma$.

The mode which $\sigma$ parametrizes is a part of the original theory which cannot be treated as free, even after localization. Therefore, the values of $\sigma_{k}$ are subject to the boundary conditions implied by the original codimension-one definition of the replica defect. It is 
trivial to see that this implies that all $\sigma_{k}$ are equal. ${ }^{16}$ Equivalently, the sewing operation for this set of moduli consists of a set of delta functions, in the matrix model for the $n$-copy theory in the presence of the defect, which enforce this equality

$$
\delta_{\text {moduli }}^{\text {scalar }}=\prod_{k=0}^{n-2} \prod_{i=1}^{\operatorname{rank} G} \delta\left(\left(\sigma_{k}\right)_{i}-\left(\sigma_{k+1}\right)_{i}\right) .
$$

The classical contributions depending only on $\sigma$ are products of expressions of the form

$$
e^{c\left(\prod_{i} \omega_{i}^{-1}\right) \operatorname{Tr}\left(\sigma^{p}\right)},
$$

for some constant $c$. In the theory considered on the squashed sphere, we have

$$
\prod \omega_{i}^{-1}=n,
$$

while for the round sphere

$$
\prod \omega_{i}^{-1}=1 .
$$

Starting from the $n$-copy theory on the round sphere, after using the delta functions to set all the $\sigma_{k}$ equal, we recover the factor of $n$.

\subsection{Perturbative contributions}

The defect operator interpretation of the supersymmetric Rényi entropy was originally observed in [6] by rewriting the perturbative partition function on the $n$-fold cover as $n$ copies of the partition functions on a round three-sphere with vortex loops inserted on each copy. (See also $[16,17]$ for related works in two dimensions). We extend this interpretation to higher dimensions and show the perturbative parts of the partition functions in the $4 d$ and $5 d$ supersymmetric Rényi entropies also have similar structures. The non-perturbative contributions arise in higher dimensions will be discussed separately in section 3.6.

First, let us review the story in three dimensions [6]. There are no non-perturbative contributions in the matrix model (3.19) and we only need to deal with the one-loop partition functions appearing as the double sine functions $S_{2}$ from the vector and matter multiplets. The identity (B.7) for the double sine function yields that the one-loop partition function of a multiplet with R-charge $\Delta_{I}$ can be decomposed as a product of those in the presence of a supersymmetric abelian vortex loop [25]

$$
\begin{aligned}
S_{2}\left(i \rho(\sigma)+\frac{|\boldsymbol{\omega}|}{2} \Delta_{I} \mid 1, \frac{1}{n}\right)^{-1} & =\prod_{k=0}^{n-1} S_{2}\left(i \rho(\sigma)+\frac{\Delta_{I}}{2}\left(1+\frac{1}{n}\right)+\frac{k}{n} \mid 1,1\right)^{-1}, \\
& =\prod_{k=0}^{n-1} S_{2}\left(i \rho(\sigma)+q_{k}^{3 d} \text { vortex }+\Delta_{I} \mid 1,1\right)^{-1}
\end{aligned}
$$

where $q_{k}^{3 d}$ vortex is introduced to be

$$
q_{k}^{3 d \text { vortex }}=\frac{\Delta_{I}}{2}\left(\frac{1}{n}-1\right)+\frac{k}{n} .
$$

\footnotetext{
${ }^{16}$ This only makes sense if one identifies the gauge transformations at the interface. This also implies the identification of the residual gauge transformations acting in the different matrix models.
} 
Comparing it with the shift of the modulus $\sigma$ (2.12), we interpret the decomposition as a manifestation of the introduction of a supersymmetric abelian vortex loop, described in section 2.2, of charge $q_{k}^{\text {vortex }}$ supported on the entangling surface $(\theta=0)$ on each copy of a round sphere. Note that $q_{k}^{\text {vortex }}$ differs from (2.12) by a term proportional to the R-charge $\Delta_{I}$ for matter multiplets. This means that the vortex loops for the supersymmetric Rényi entropy are dressed by the R-symmetry flux.

One can work out a similar decomposition for the perturbative part in the $4 d \mathcal{N}=2$ matrix model (3.21) with a slight modification. The matter one-loop partition function is represented by the $\Upsilon$ function, which enjoys decomposition into the product of the $n$-copies with the help of the identity (B.12):

$$
\Upsilon\left(i \rho(a)+i m+\frac{|\boldsymbol{\omega}|}{2} \mid 1, \frac{1}{n}\right)^{-1}=\prod_{k=0}^{n-1} \Upsilon\left(i \rho(a)+1+q_{k}^{\text {vortex }} \mid 1,1\right)^{-1}
$$

where $q_{k}^{\text {vortex }}$ is to be interpreted as the charge of a codimension-two surface defect

$$
q_{k}^{\text {vortex }}=\frac{k}{n}
$$

under the choice of the shifted mass (3.14) and the relation (A.5). It agrees with the shift of the modulus (2.60) induced by the insertion of a supersymmetric abelian surface operator of charge $k$ in section 2.3 .

Repeating the analogous procedure to the $5 d \mathcal{N}=1$ theory one finds the matter one-loop partition function with the identity (B.7):

$$
S_{3}\left(i \rho(\sigma)+i m+\frac{|\boldsymbol{\omega}|}{2} \mid 1,1, \frac{1}{n}\right)^{-1}=\prod_{k=0}^{n-1} S_{3}\left(i \rho(\sigma)+1+q_{k}^{\text {vortex }} \mid 1,1,1\right)^{-1}
$$

where we introduce the charges $q_{k}^{\text {vortex }}$ by (3.34) and the mass shift (3.16). Once again it can be interpreted as a supersymmetric codimension-two surface defect of charge $k$ described in section 2.4.

It is straightforward to apply the same argument to vector multiplets in any dimensions to read off the surface charges and the results (3.32) and (3.34) still hold with $\Delta_{I}=0$.

\subsection{Non-perturbative contributions — instantons and contact-instantons}

The partition functions on the four-sphere and the five-sphere receive non-perturbative contributions from instantons and contact-instantons, respectively. These are supersymmetric configurations localized at fixed loci of the equivariant action generated by the square of the supersymmetry. For the four-sphere, the fixed points are at the north and south pole and give rise to instanton and anti-instanton contributions, respectively. The five-sphere partition function includes contributions from contact instantons [43], which are extended along the fiber of

$$
\mathbb{S}^{1} \rightarrow \mathbb{S}^{5} \rightarrow \mathbb{C} P^{2}
$$




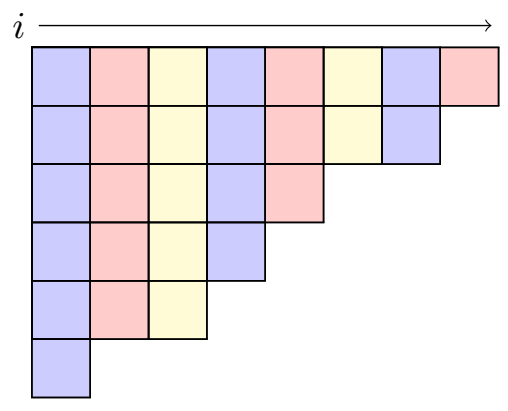

$Y_{l i}$

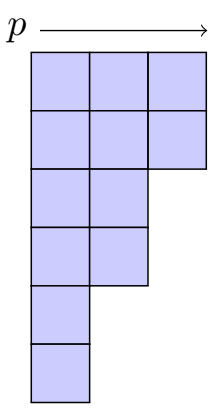

$k=0$

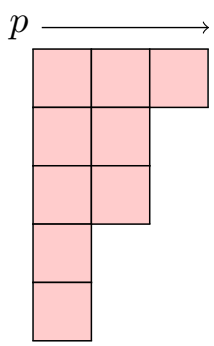

$k=1$

$Y_{l p}$

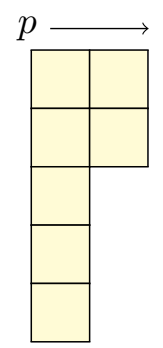

$k=2$

Figure 1. Fractionalization and recombination of the Young diagrams representing singular instantons for $n=3$.

and localized at three points on the base. ${ }^{17}$ The supersymmetric instanton contributions are computed by the Nekrasov partition function $[52,53]$. We find it convenient to express both types of contributions in terms of the $5 d$, or $q$-deformed, version of the Nekrasov partition function, which we review in appendix $\mathrm{C}$. The $4 d$ undeformed partition function can be recovered by taking an appropriate limit.

Instanton and contact-instanton contributions introduce new moduli, classical contributions, and perturbative contributions into the calculation. We begin by discussing how the moduli of the replicas in the $n$-copy theory are sewn up to produce those of the original theory. We assert a specific pattern for the fractionalization of a particular instanton configuration, given by a vector of Young diagrams. We then show that, given this pattern, the classical and perturbative contributions recombine to yield (3.4).

Similar decompositions of partitions appear in the context of instantons on ALE spaces (cf. [54]). A relationship between the instanton partition function on a $\mathbb{Z}_{n}$ orbifold of $\mathbb{C}^{2}$, acting on just one $\mathbb{C}$ factor, and surface operators appears in $[55,56]$ (see also the review [57]). It is possible that the results we need for the covering space associated to the $n$-copy theory can be recovered from the latter papers. Specifically, this seems plausible given the connection between the supersymmetric Rényi entropy at $n$ and at $1 / n$. However, we need $5 d$ results and a very specific surface operator, so we derive the necessary fractionalization and relationships between the determinants in this context.

\subsubsection{Sewing of instantons}

After employing equivariant localization, the instanton moduli space localizes to a set of points, each given by a vector of partitions $[52,53]$. The partitions describing the instanton moduli space of the theory on the branched sphere fractionalize to yield partitions for each

\footnotetext{
${ }^{17}$ To the best of our understanding, the precise form of these contributions is still conjectural. We will use the form considered in e.g. [50] and find that it works well.
} 
of the replicas in the $n$-copy theory. This happens by splitting one set of Young diagrams, yielding a partition vector $\overrightarrow{\mathbf{Y}}$, into $n$ Young diagrams, yielding partitions $\{\overrightarrow{\mathbf{Y}}(k)\}_{k=0}^{n-1}$, having the same combined number of boxes. This process is illustrated in figure 1. The diagrams can be split along the vertical or along the horizontal, depending on which deformation parameter $\epsilon_{i}$ is taken to be $n$ times smaller. Since the instanton partition function is invariant under the simultaneous transposition of the diagrams and $\epsilon_{1} \leftrightarrow \epsilon_{2}$, it suffices to consider the situation in figure 1.

For the contribution of a hypermultiplet in the fundamental representation, each element of the fluctuation determinant around an instanton can be associated to a box in the Young diagram, and figure 1 describes the decomposition into replicas. The contribution from a vector multiplet, or from an adjoint hypermultiplet, involves pairs of partitions in the vector $\overrightarrow{\mathbf{Y}}$. We find that the decomposition into replicas follows the pattern

$$
\left.\left(\mathbf{Y}_{1}, \mathbf{Y}_{2}\right) \rightarrow \prod_{k_{1,2}=0}^{n-1}\left(\mathbf{Y}_{1}\left(k_{1}\right), \mathbf{Y}_{2}\left(k_{2}\right)\right)\right|_{k_{1}-k_{2}=k \bmod n}
$$

The moduli space of contact-instantons can also fractionalize along the additional $\mathbb{S}^{1}$ direction. This process is simpler, amounting to decomposing the Kaluza-Klein (KK) momentum, and is described in section 3.6.5. It does not involve the partitions.

\subsubsection{Classical and Chern-Simons contributions depending on instantons}

The classical weight in the instanton partition function of a configuration with instanton number $|\overrightarrow{\mathbf{Y}}|$ in a $5 d$ SCFT is

$$
\mathfrak{q}^{|\vec{Y}|}
$$

The number of boxes in the Young diagrams on the right pane of figure 1 sum to the number of boxes on the left, and therefore the combined weight from each of the replicas matches that of the original theory

$$
\mathfrak{q}^{|\overrightarrow{\mathbf{Y}}|}=\prod_{k=0}^{n-1} \mathfrak{q}^{|\overrightarrow{\mathbf{Y}}(k)|}
$$

In the presence of a $5 d$ Chern-Simons term, we also need to split the contribution

$$
Z_{\overrightarrow{\mathbf{Y}}, \kappa}^{5 d-\mathrm{CS}}\left(\vec{a}, \epsilon_{1}, \epsilon_{2}, \beta\right)=\exp \left[i \beta \kappa \sum_{l} \sum_{(s, t) \in \mathbf{Y}_{l}}\left(a_{l}-(s-1) \epsilon_{1}-(t-1) \epsilon_{2}\right)\right] .
$$

We do this first for the case where $\epsilon_{1}=1 / n$ by setting $s=n p-k$

$$
\begin{aligned}
Z_{\overrightarrow{\mathbf{Y}}, \kappa}^{5 d-\mathrm{CS}}\left(\vec{a}, \frac{1}{n}, 1,2 \pi\right) & =\exp \left[2 \pi i \kappa \sum_{l} \sum_{(s, t) \in \mathbf{Y}_{l}}\left(a_{l}-\frac{s-1}{n}-(t-1)\right)\right], \\
& =\prod_{k=0}^{n-1} \exp \left[2 \pi i \kappa \sum_{l} \sum_{(p, t) \in \mathbf{Y}_{l}(k)}\left(a_{l}-\frac{k}{n}-(p-1)-(t-1)\right)\right],
\end{aligned}
$$


where in the second line we have reparametrized

$$
k \rightarrow n-1-k .
$$

The expression on the second line can be thought of as the contributions from the $n$-replicas, where the additional shift

$$
a_{l} \rightarrow a_{l}-\frac{k}{n},
$$

is the effect of the monodromy brought on by the defect. Since we associate this shift with

$$
q_{k}^{\text {surface }}=\frac{k}{n},
$$

we see that the contribution from the Higgs type mass enters the Chern-Simons term in the same way as it enters the fluctuation determinant for the fundamental hypermultiplet considered below.

The contribution from the $k^{\text {th }}$ replica is sensitive only to the partition represented by the Young diagrams $\overrightarrow{\mathbf{Y}}(k)$. It might seem strange to see the mass shift appear at all in a classical contribution. Note, however, that the combination appearing in the exponential comes from evaluating the classical Chern-Simons term at the positions of the poles for the integral over the scalar associated to the auxiliary $U(|\overrightarrow{\mathbf{Y}}|)$ symmetry [58-60]. This position is shifted by the Higgs type mass term.

\subsubsection{Fundamental hypermultiplets fluctuations}

We now demonstrate the relationship between the contribution of a hypermultiplet on the squashed sphere, with deformation parameters $\left(\epsilon_{1}, \epsilon_{2}, \beta\right)=\left(\frac{1}{n}, 1,2 \pi\right)$, and $n$ hypermultiplets in the presence of defects with deformation parameters $\left(\epsilon_{1}, \epsilon_{2}, \beta\right)=(1,1,2 \pi)$. At the conformal point

$$
Q_{m_{f}^{0}}^{-1}=t
$$

Expressing the squashed sphere contribution using the round sphere values for $t, q$ etc., we get

$$
\begin{aligned}
Z_{\overrightarrow{\mathbf{Y}}}^{\text {fund hyper }}\left(\vec{a}, m_{f}^{0}, \frac{1}{n}, 1,2 \pi\right) & =\prod_{l=1}^{N} \prod_{j=1}^{\infty} \frac{\left(Q_{l} q t^{-\frac{j-1}{n}} ; q\right)_{\infty}}{\left(Q_{l} q^{Y_{l j}+1} t^{-\frac{j-1}{n}} ; q\right)_{\infty}}, \\
& =\prod_{k=0}^{n-1}\left[\prod_{l=1}^{N} \prod_{j=1}^{\infty} \frac{\left(Q_{l} q t^{-\frac{j-1}{n}} ; q\right)_{\infty}}{\left(Q_{l} q^{Y_{l j}+1} t^{-\frac{j-1}{n}} ; q\right)_{\infty}}\right]_{j=n p-k}, \\
& =\prod_{k=0}^{n-1}\left[\prod_{l=1}^{N} \prod_{p=1}^{\infty} \frac{\left(Q_{m_{f}}^{-1}(k) Q_{l} q t^{-(p-1)} ; q\right)_{\infty}}{\left(Q_{m_{f}}^{-1}(k) Q_{l} q^{Y_{l, n p-k}+1} t^{-(p-1)} ; q\right)_{\infty}}\right],
\end{aligned}
$$

and

$$
Q_{m_{f}}^{-1}(k) \equiv t^{\frac{k}{n}-1+\frac{1}{n}}
$$


Reparametrizing the product over $k$ as $k \rightarrow n-1-k$, we get

$$
Q_{m_{f}}^{-1}(k) \equiv t^{-\frac{k}{n}}
$$

implying

$$
m_{f}=\frac{k}{n}
$$

We now identify the terms in the square parentheses with the contribution of fluctuations of a hypermultiplet in the presence of a codimension-two defect

$$
Z_{\overrightarrow{\mathbf{Y}}}^{\text {hyper-defect }}(\vec{a}, 1,1,2 \pi ; k) \equiv\left[\prod_{l=1}^{N} \prod_{p=1}^{\infty} \frac{\left(Q_{m_{f}}^{-1}(k) Q_{l} q t^{-(p+1)} ; q\right)_{\infty}}{\left(Q_{m_{f}}^{-1}(k) Q_{l} q^{Y_{l, n p+k}+1} t^{-(p+1)} ; q\right)_{\infty}}\right],
$$

The expression for $Z_{\overrightarrow{\mathbf{Y}}}^{\text {hyper-defect }}(k)$ differs from the expression $Z_{\overrightarrow{\mathbf{Y}}}^{\text {hyper }}$ in two ways

1. The fugacity, or mass parameter, involving the background vector is shifted in the imaginary direction by

$$
q_{\mathrm{vortex}}=\frac{k}{n} .
$$

We ascribe this to the effect of the codimension-two defect on the fluctuations.

2. The $k^{\text {th }}$ such contribution is sensitive only to the boxes of the Young diagram with horizontal position given by

$$
i=k \bmod n .
$$

We ascribe this to the fractionalization of the instanton moduli corresponding to the partition.

These are the same effects visible for the Chern-Simons contribution. We conclude that

$$
Z_{\overrightarrow{\mathbf{Y}}}^{\text {fund hyper }}\left(\vec{a}, m_{f}^{0}, \frac{1}{n}, 1,2 \pi\right)=\prod_{k=0}^{n-1} Z_{\overrightarrow{\mathbf{Y}}}^{\text {hyper-defect }}(\vec{a}, 1,1,2 \pi ; k) .
$$

\subsubsection{Adjoint hypermultiplet or vector multiplet fluctuations}

An adjoint hypermultiplet contributes to the fluctuation determinant around an instanton configuration as

$$
\begin{aligned}
Z_{\overrightarrow{\mathbf{Y}}}^{\text {adjoint hyper }}\left(\vec{a}, m_{f}^{0}, \epsilon_{1}, \epsilon_{2}, \beta\right)= & \prod_{(l, i) \neq(m, j)} \frac{\left(Q_{m_{f}^{0}} Q_{l} Q_{m}^{-1} q^{Y_{l i}-Y_{m j}} t^{j-i+1} ; q\right)_{\infty}}{\left(Q_{m_{f}^{0}}^{-1} Q_{l} Q_{m}^{-1} q^{Y_{l i}-Y_{m j} t^{j-i} ; q}\right)_{\infty}} \\
& \cdot \frac{\left(Q_{m_{f}^{0}}^{-1} Q_{l} Q_{m}^{-1} t^{j-i} ; q\right)_{\infty}}{\left(Q_{m_{f}^{0}} Q_{l} Q_{m}^{-1} t^{j-i+1} ; q\right)_{\infty}}
\end{aligned}
$$

This involves pairs of partitions $Y_{l}$ and $Y_{m}$. The virtue of the form of the fluctuation determinant written above is that $t$ appears raised only to a power corresponding the 
column indices $i, j$. As such, the determinant can be decomposed in a way similar to the fundamental hypermultiplet

$$
\begin{aligned}
& Z_{\overrightarrow{\mathbf{Y}}}^{\text {adjoint hyper }}\left(\vec{a}, \frac{1}{n}, 1,1,2 \pi\right) \\
& \quad=\prod_{(l, i),(m, j)} \frac{\left(Q_{l} Q_{m}^{-1} q^{Y_{l i}-Y_{m j}} t^{\frac{1}{n}(j-i)} ; q\right)_{\infty}}{\left(Q_{l} Q_{m}^{-1} q^{Y_{l i}-Y_{m j}} t^{\frac{1}{n}(j-i+1)} ; q\right)_{\infty}} \frac{\left(Q_{l} Q_{m}^{-1} t^{\frac{1}{n}(j-i+1)} ; q\right)_{\infty}}{\left(Q_{l} Q_{m}^{-1} t^{\frac{1}{n}(j-i)} ; q\right)_{\infty}}, \\
& =\prod_{k=0}^{n-1}\left[\prod_{(l, i),(m, j)}^{j-i=n(p-q)+k} \frac{\left(Q_{l} Q_{m}^{-1} q^{Y_{l i}-Y_{m j}} t^{p-q+\frac{k}{n}} ; q\right)_{\infty}}{\left(Q_{l} Q_{m}^{-1} q^{Y_{m j}-Y_{l i}} t^{p-q+1-\frac{k}{n}} ; q\right)_{\infty}} \frac{\left(Q_{l} Q_{m}^{-1} t^{p-q+1-\frac{k}{n}} ; q\right)_{\infty}}{\left(Q_{l} Q_{m}^{-1} t^{p-q+\frac{k}{n}} ; q\right)_{\infty}}\right], \\
& =\prod_{k=0}^{n-1}\left[\prod_{(l, i),(m, j)}^{i-j=n(p-q)+k} \frac{\left(Q_{m_{f}}(k) Q_{l} Q_{m}^{-1} q^{Y_{l i}-Y_{m j}} t^{p-q} ; q\right)_{\infty}}{\left(Q_{m_{f}}^{-1}(k) Q_{l} Q_{m}^{-1} q^{Y_{m j}-Y_{l i}} t^{p-q+1} ; q\right)_{\infty}} \frac{\left(Q_{m_{f}}^{-1}(k) Q_{l} Q_{m}^{-1} t^{p-q+1} ; q\right)_{\infty}}{\left(Q_{m_{f}}(k) Q_{l} Q_{m}^{-1} t^{p-q} ; q\right)_{\infty}}\right] .
\end{aligned}
$$

In the second line, we have replaced in two of the factors $k \rightarrow n-1-k$. If we define

$$
\begin{aligned}
& Z_{\overrightarrow{\mathbf{Y}}}^{\text {adjoint -defect }}(\vec{a}, 1,1,2 \pi ; k) \\
& \quad \equiv \prod_{(l, i),(m, j)}^{i-j=n(p-q)+k} \frac{\left(Q_{m_{f}}(k) Q_{l} Q_{m}^{-1} q^{Y_{l i}-Y_{m j}} t^{p-q} ; q\right)_{\infty}}{\left(Q_{m_{f}}^{-1}(k) Q_{l} Q_{m}^{-1} q^{Y_{m j}-Y_{l i}} t^{p-q+1} ; q\right)_{\infty}} \frac{\left(Q_{m_{f}}^{-1}(k) Q_{l} Q_{m}^{-1} t^{p-q+1} ; q\right)_{\infty}}{\left(Q_{m_{f}}(k) Q_{l} Q_{m}^{-1} t^{p-q} ; q\right)_{\infty}},
\end{aligned}
$$

such that

$$
Z_{\overrightarrow{\mathbf{Y}}}^{\text {adjoint hyper }}\left(\vec{a}, m_{f}^{0}, \frac{1}{n}, 1,2 \pi\right)=\prod_{k=0}^{n-1} Z_{\overrightarrow{\mathbf{Y}}}^{\text {adjoint-defect }}(\vec{a}, 1,1,2 \pi ; k) \text {. }
$$

We now consider the contribution of a vector multiplet. Its fluctuation determinant is inverse to that of an adjoint hypermultiplet with zero mass, as was the case for the perturbative contribution. As we did there, we keep a "mass fugacity" to keep track of the deformation brought on by the defect. Since this mass is now associated to the Higgs vev, it is not shifted from $m_{f}=0$ by the Okuda-Pestun prescription. Repeating the calculation for the adjoint hypermultiplet using the same manipulations, we get

$$
Z_{\overrightarrow{\mathbf{Y}}}^{\text {vector }}\left(\vec{a}, m_{f}^{0}, \frac{1}{n}, 1,2 \pi\right)=\prod_{k=0}^{n-1} Z_{\overrightarrow{\mathbf{Y}}}^{\text {vector-defect }}(\vec{a}, 1,1,2 \pi ; k),
$$

with

$$
\begin{aligned}
& Z_{\overrightarrow{\mathbf{Y}}}^{\text {vector-defect }}(\vec{a}, 1,1,2 \pi ; k) \\
& \quad \equiv \prod_{(l, i) \neq(m, j)}^{i-j=n(p-q)+k} \frac{\left(Q_{m_{f}}(k) Q_{l} Q_{m}^{-1} q^{Y_{l i}-Y_{m j}} t^{p-q} ; q\right)_{\infty}}{\left(Q_{m_{f}}^{-1}(k) Q_{l} Q_{m}^{-1} q^{Y_{m j}-Y_{l i}} t^{p-q+1} ; q\right)_{\infty}} \frac{\left(Q_{m_{f}}^{-1}(k) Q_{l} Q_{m}^{-1} t^{p-q+1} ; q\right)_{\infty}}{\left(Q_{m_{f}}(k) Q_{l} Q_{m}^{-1} t^{p-q} ; q\right)_{\infty}}
\end{aligned}
$$

Since the original product is over pairs of Young diagrams corresponding to $l$ and $m$, the decomposition of an adjoint hypermultiplet or a vector multiplet into $n$ parts is not as simple as in figure 1. Instead, each pair of diagrams of the theory on the squashed sphere splits into $n$ pairs for each of the $n$ copies. 


\subsubsection{The third point}

The $5 d$ squashed sphere has one more contribution, not of the type above. The third point contributes a sum over contact-instantons with deformation parameters $\left(\epsilon_{1}, \epsilon_{2}, \beta\right)=$ $(1,1,2 \pi n)$, i.e. $\beta$ is $n$ times as large as it would be on the round sphere. In order to decompose this contribution, it is useful to write the determinant part of the $q$-deformed instanton partition function as a product over Kaluza Klein modes coming from the extra circle. Starting from the expressions in [61]

$$
Z_{\overrightarrow{\mathbf{Y}}}^{\text {vector }}\left(\vec{a}, \epsilon_{1}, \epsilon_{2}, \beta\right)=\prod_{l, m}^{N_{c}}\left(N_{l, m}^{\overrightarrow{\mathbf{Y}}}\right)^{-1},
$$

where

$$
N_{l, m}^{\overrightarrow{\mathbf{Y}}}=\prod_{s \in Y_{l}}\left[1-e^{i \beta\left(\ell_{Y_{m}}(s) \epsilon_{1}-\left(a_{Y_{l}}(s)+1\right) \epsilon_{2}+a_{l}-a_{m}\right)}\right] \prod_{t \in Y_{m}}\left[1-e^{i \beta\left(-\left(\ell_{Y_{l}}(t)+1\right) \epsilon_{1}+a_{Y_{m}}(t) \epsilon_{2}+a_{l}-a_{m}\right)}\right],
$$

the defect decomposition, in this case, follows simply from the identity

$$
\prod_{k=0}^{n-1}\left[1-e^{2 \pi i\left(\alpha+\frac{k}{n}\right)}\right]=1-e^{2 \pi i n \alpha} .
$$

A similar expression exists for the hypermultiplet contribution. Its decomposition follows from the same method. ${ }^{18}$

To relate this decomposition to the KK decomposition, we use the regularized infinite product

$$
\prod_{m=-\infty}^{\infty}(m+a)=1-e^{2 \pi i a}, \quad \operatorname{Im}(a)>0 .
$$

The defect decomposition can now be thought of as writing the quantum number $m$ in the form

$$
m=n p+k .
$$

The partitions of the various copies are simply identified in this case, in analogy with the vev of the scalar modulus.

\section{$3.7 n \rightarrow 1 / n$ duality}

The supersymmetric Rényi entropy in three and four dimensions satisfies an interesting property stemming from the fact that for a superconformal theory

$$
Z_{n}^{3 d \text { or } 4 d}=Z_{1 / n}^{3 d \text { or } 4 d} .
$$

This follows simply from two facts:

1. Conformal invariance implies a dependence on the squashing parameters $\omega_{1 / 2}$ of the form

$$
Z^{3 d \text { or } 4 d}\left(\frac{\omega_{1}}{\omega_{2}}\right) \text {. }
$$

\footnotetext{
${ }^{18}$ The mass shift given by $m_{f}^{0}$ is immaterial in this case.
} 
2. There is a trivial change of coordinates which exchanges

$$
\omega_{1} \leftrightarrow \omega_{2}
$$

Taking $\omega_{1}=1 / n$ and $\omega_{2}=1$ yields (3.65). The same trick does not work in five dimensions. This relationship can be thought of as an interacting supersymmetric version of the BoseFermi duality in three dimensions [62], which does not hold for Rényi entropies in higher dimensions without introducing supersymmetry.

One can calculate the $n^{\text {th }}$ supersymmetric Rényi entropy from the partition function on the branched sphere. From the point of view of the instanton partition function, taking

$$
\epsilon_{1}=1 / n, \quad \epsilon_{2}=1,
$$

corresponds to counting instantons on a space which is branched over a codimension-two surface. Taking

$$
\epsilon_{1}=n, \quad \epsilon_{2}=1,
$$

on the other hand, corresponds to counting instantons on an orbifold. It is interesting that the two counts are related.

\section{Discussion}

We have shown that the supersymmetric Rényi entropy (SRE) can be computed using supersymmetric codimension-two defects. After giving a microscopic definition of the defect operators, we computed the expectation values of these defects using localization. ${ }^{19}$ We showed that the effect of such defects on the matrix models calculating the partition function on the round sphere amounted to imaginary mass terms. We made a conjecture regarding the details of the sewing operation needed to complete the picture for the moduli, scalar vevs, instantons and contact-instantons, encountered in localization. We then showed the equality with the squashed sphere partition function.

Although we explicitly only showed agreement of the partition functions representing the SRE, the decomposition into defects seems to work at the level of the matrix model ingredients, and for any deformation parameters $\omega_{i}$. It is reasonable to conjecture that it works at the level of the $5 d$ holomorphic blocks and gluing [50,63, 64]. If this is the case, a relationship similar to the one described here should hold for the partition functions on four-manifolds and five-manifolds of the type described in e.g. [65, 66].

In the context of holographic duality, the Ryu-Takanayagi prescription [67, 68] allows us to compute the entanglement entropy in a CFT, in a particular limit corresponding to classical gravity in the bulk, using a minimal area surface in AdS which is homologous to a given entangling region $\Sigma$. Corrections to this computation have recently been conjectured in $[69,70]$. A variant for the Rényi entropy was put forth in [71]. Somewhat similar

\footnotetext{
${ }^{19}$ The supersymmetric codimension-two defects coincide, in three and in four dimensions, with specific versions of the operators defined in [24] and [35] respectively. Five-dimensional versions were considered in e.g. [40].
} 
prescriptions are used to compute the expectation values of supersymmetric non-local operators (see e.g. [72-78]). The authors, and others, have long suspected that there is a relationship between these computations. We do not, however, know of a concrete example of such a relationship. We hope that the definition of the supersymmetric defect operator version of the SRE calculation can be used to find one. This may involve going, first, to a dual picture in the SCFT. For instance, the codimension-two defects realizing the SRE in a $3 d \mathcal{N}=2$ theory are vortex loops, which, in certain situations, are dual to a Wilson loop under $3 d$ mirror symmetry [24].

\section{Acknowledgments}

We would like to thank S. Hellerman, C. Herzog, K. Hosomichi, D. Jafferis, R. Myers, T. Okuda, A. Sheshmani, Y. Tachikawa and B. Willett for valuable discussions. The work of T.N. was supported in part by JSPS Grant-in-Aid for Young Scientists (B) No. 15K17628 and JSPS Grant-in-Aid for Scientific Research (A) No.16H02182. The work of I.Y. was supported by World Premier International Research Center Initiative (WPI), MEXT, Japan.

\section{A Conventions}

We summarize our conventions for gauge theories and the matrix models resulting from the localization procedure in three, four, and five dimensions. To begin with, we set an overall scale associated with the size of $\mathbb{S}^{3,4,5}$

$$
\ell=1 \text {. }
$$

Dimensionful parameters such as $\epsilon_{1,2}, \omega_{1,2,3}, a, m, \beta$ etc. are expressed using this scale.

We use physics conventions for the gauge and flavor symmetry groups. The generators of the Lie algebra $u(N)$ are taken to be Hermitian matrices, and factors of $i$ appear in appropriate places in the field strength. Consequently, integration over the Cartan subalgebra means an $N$-dimensional real integral over variables denoted $\vec{\sigma}$, which are the eigenvalues of a matrix $\sigma$. In $5 d, \vec{\sigma}$ is related to the scalar vev as

$$
\langle\phi\rangle=i \sigma .
$$

In $4 d$ we have

$$
\langle\phi\rangle=\langle\bar{\phi}\rangle=-i \frac{\sigma}{2} .
$$

In $3 d$, where the real adjoint scalar in the vector multiplet is also denoted $\sigma$, we have

$$
\langle\sigma\rangle=\sigma .
$$

This convention extends to mass parameters, which are vevs for scalars in background vector multiplets. The physical mass of a chiral multiplet or hypermultiplet is a real number $m$, which bears the same relation to the background vev as $\sigma$ does to the dynamical 
vev. The deformation parameters $a$ and $m_{f}$, which are used when discussing the instanton contributions to the partition function in $4 d$ and $5 d$, are set to

$$
a=i \sigma, \quad m_{f}=i m .
$$

Our conventions for spinors and supersymmetry transformations are different in different dimensions. However, supersymmetry transformation parameters are always taken to be commuting spinors.

\section{B Special functions}

We summarize the definitions and identities for the special functions appear in the text.

Multiple gamma function. For $\boldsymbol{\omega}=\left(\omega_{1}, \cdots, \omega_{r}\right) \geq 0$ and $z \in \mathbb{C}$, the multiple Hurwitz zeta function is defined by

$$
\zeta_{r}(s, z, \boldsymbol{\omega}):=\sum_{\boldsymbol{n} \geq 0}(\boldsymbol{n} \cdot \boldsymbol{\omega}+z)^{-s},
$$

where $\boldsymbol{n}=\left(n_{1}, \cdots, n_{r}\right) \geq 0$. The integral representation is

$$
\zeta_{r}(s, z, \boldsymbol{\omega})=\frac{1}{\Gamma(s)} \int_{0}^{\infty} \frac{e^{-z t}}{\prod_{i=1}^{r}\left(1-e^{-\omega_{i} t}\right)} t^{s-1} d t .
$$

For an integer $N$, one can prove the identity

$$
\zeta_{r}\left(s, z, \omega_{1}, \cdots, \omega_{r-1}, \frac{\omega_{r}}{N}\right)=\sum_{k=0}^{N-1} \zeta_{r}\left(s, z+\frac{k \omega_{r}}{N}, \boldsymbol{\omega}\right) .
$$

The Barnes multiple gamma function $\Gamma_{r}(z \mid \boldsymbol{\omega})$ is defined by

$$
\Gamma_{r}(z \mid \boldsymbol{\omega}):=\exp \left[\left.\partial_{s} \zeta_{r}(s, z, \boldsymbol{\omega})\right|_{s=0}\right] .
$$

Multiple sine function. One can define the $r$-ple sine function $S_{r}(z \mid \boldsymbol{\omega})$ by

$$
S_{r}(z \mid \boldsymbol{\omega}):=\Gamma_{r}(z \mid \boldsymbol{\omega})^{-1} \Gamma_{r}(|\boldsymbol{\omega}|-z \mid \boldsymbol{\omega})^{(-1)^{r}},
$$

with $|\boldsymbol{\omega}|=\sum_{i=1}^{r} \omega_{i}$. It satisfies the following identities [79]:

$$
\begin{aligned}
S_{r}(|\boldsymbol{\omega}|-z \mid \boldsymbol{\omega}) & =S_{r}(z \mid \boldsymbol{\omega})^{(-1)^{r-1}}, \\
S_{r}\left(z+\omega_{i} \mid \boldsymbol{\omega}\right) & =S_{r}(z \mid \boldsymbol{\omega}) S_{r-1}(z, \boldsymbol{\omega}(i))^{-1}, \\
S_{r}(N z \mid \boldsymbol{\omega}) & =\prod_{0 \leq k_{i} \leq N-1} S_{r}\left(z+\frac{\boldsymbol{k} \cdot \boldsymbol{\omega}}{N} \mid \boldsymbol{\omega}\right), \\
N & =\prod_{0 \leq k_{i} \leq N-1, \boldsymbol{k} \neq 0} S_{r}\left(\frac{\boldsymbol{k} \cdot \boldsymbol{\omega}}{N} \mid \boldsymbol{\omega}\right), \\
S_{r}(c z \mid c \boldsymbol{\omega}) & =S_{r}(z \mid \boldsymbol{\omega}), \text { for } \quad c>0 .
\end{aligned}
$$

where $\boldsymbol{\omega}(i)=\left(\omega_{1}, \cdots, \omega_{i-1}, \omega_{i+1}, \cdots, \omega_{r}\right)$. 
The formula (B.3) yields an additional identity

$$
S_{r}\left(z \mid \omega_{1}, \cdots, \omega_{r-1}, \frac{\omega_{r}}{N}\right)=\prod_{k=0}^{N-1} S_{r}\left(z+\frac{k \omega_{r}}{N} \mid \boldsymbol{\omega}\right) .
$$

This is the generalization of the identity for the hyperbolic gamma function found in [6].

The $\Upsilon$ function. The double gamma function is used to define the $\Upsilon$ function $[29,80]$

$$
\Upsilon\left(z \mid \omega_{1}, \omega_{2}\right):=\Gamma_{2}^{2}\left(\frac{|\boldsymbol{\omega}|}{2} \mid \omega_{1}, \omega_{2}\right)\left(\Gamma_{2}\left(z \mid \omega_{1}, \omega_{2}\right) \Gamma_{2}\left(|\boldsymbol{\omega}|-z \mid \omega_{1}, \omega_{2}\right)\right)^{-1}
$$

satisfies several identities

$$
\begin{aligned}
& \Upsilon\left(z+\omega_{1} \mid \omega_{1}, \omega_{2}\right)=\omega_{2}^{\frac{2 z}{\omega_{2}}-1} \gamma\left(z / \omega_{2}\right) \Upsilon\left(z \mid \omega_{1}, \omega_{2}\right), \\
& \Upsilon\left(z+\omega_{2} \mid \omega_{1}, \omega_{2}\right)=\omega_{1}^{\frac{2 z}{\omega_{1}}-1} \gamma\left(z / \omega_{1}\right) \Upsilon\left(z \mid \omega_{1}, \omega_{2}\right),
\end{aligned}
$$

where $\gamma(z):=\Gamma(z) / \Gamma(1-z)$ and the scaling law

$$
\Upsilon\left(c z \mid c \omega_{1}, c \omega_{2}\right)=c^{\frac{(|\boldsymbol{\omega}|-2 z)^{2}}{4 \omega_{1} \omega_{2}}} \Upsilon\left(z \mid \omega_{1}, \omega_{2}\right) .
$$

Some literatures including $[29,80]$ use

$$
\Upsilon_{b}(z):=\Upsilon(z \mid b, 1 / b) .
$$

which is sometimes denoted $\Upsilon(z)$ without the subscript.

The formula (B.3) yields

$$
\Upsilon\left(z \mid \omega_{1}, \frac{\omega_{2}}{N}\right)=\prod_{k=0}^{N-1} \Upsilon\left(z+\frac{k \omega_{2}}{N} \mid \omega_{1}, \omega_{2}\right) .
$$

\section{The instanton partition function}

Nekrasov's instanton partition function, [52, 53], is the equivariant volume of the instanton moduli space with respect to the action of

$$
\mathrm{U}(1)_{a} \times \mathrm{U}(1)_{\epsilon_{1}} \times \mathrm{U}(1)_{\epsilon_{2}} .
$$

The three factors correspond to (constant) gauge transformations and to rotations in two orthogonal two-planes inside $\mathbb{R}^{4}$, respectively. The $q$-deformed version of the partition function counts instantons extended along an additional $\mathbb{S}^{1}$ factor in the geometry of circumference $\beta$. The undeformed partition function can be recovered by letting the size of this $\mathbb{S}^{1}$ shrink to 0 . Our expressions for the instanton partition function are taken from [61]. We use a $5 d$ parameter $\beta$ which can be used to take the $4 d$ limit, and our conventions differ from those in [61] by the substitutions

$$
\epsilon_{1} \rightarrow i \beta \epsilon_{1}, \quad \epsilon_{2} \rightarrow i \beta \epsilon_{2}, \quad a \rightarrow i \beta a .
$$


The $q$-deformed version of the instanton partition function for $G=\mathrm{U}(N)$ and in the presence of hypermultiplets can be expressed as follows [61, 81]

$$
\begin{aligned}
Z_{\text {inst }}\left(\mathfrak{q}, \vec{a}, \vec{m}_{f}, \epsilon_{1}, \epsilon_{2}, \beta\right) & =\sum_{\overrightarrow{\mathbf{Y}}} \mathfrak{q} \mid \overrightarrow{\mathbf{Y}} Z_{\overrightarrow{\mathbf{Y}}, \kappa}^{5 d-\mathrm{CS}}\left(\vec{a}, \epsilon_{1}, \epsilon_{2}, \beta\right) Z_{\overrightarrow{\mathbf{Y}}}\left(\vec{a}, \vec{m}_{f}, \epsilon_{1}, \epsilon_{2}, \beta\right), \\
Z_{\overrightarrow{\mathbf{Y}}}\left(\vec{a}, \vec{m}_{f}, \epsilon_{1}, \epsilon_{2}, \beta\right) & =Z_{\overrightarrow{\mathbf{Y}}}^{\text {vector }}\left(\vec{a}, \epsilon_{1}, \epsilon_{2}, \beta\right) \prod_{l=1}^{N_{f}} Z_{\overrightarrow{\mathbf{Y}}}^{\text {hyper }}\left(\vec{a},\left(m_{f}\right)_{l}, \epsilon_{1}, \epsilon_{2}, \beta\right), \\
Z_{\overrightarrow{\mathbf{Y}}}^{\text {vector }}\left(\vec{a}, \epsilon_{1}, \epsilon_{2}, \beta\right) & =\prod_{(l, i) \neq(m, j)} \frac{\left(Q_{l} Q_{m}^{-1} q^{Y_{l i}-Y_{m j}} t^{j-i} ; q\right)_{\infty}}{\left(Q_{l} Q_{m}^{-1} q^{Y_{l i}-Y_{m j}} t^{j-i+1} ; q\right)_{\infty}} \frac{\left(Q_{l} Q_{m}^{-1} t^{j-i+1} ; q\right)_{\infty}}{\left(Q_{l} Q_{m}^{-1} t^{j-i} ; q\right)_{\infty}}, \quad(\mathrm{C} .4) \\
Z_{\overrightarrow{\mathbf{Y}}}^{\text {adjoint hyper }}\left(\vec{a}, m_{f}, \epsilon_{1}, \epsilon_{2}, \beta\right) & =\prod_{(l, i),(m, j)} \frac{\left(Q_{m_{f}} Q_{l} Q_{m}^{-1} q^{Y_{l i}-Y_{m j}} t^{j-i+1} ; q\right)_{\infty}}{\left(Q_{m_{f}}^{-1} Q_{l} Q_{m}^{-1} q^{Y_{l i}-Y_{m j}} t^{j-i} ; q\right)_{\infty}} \frac{\left(Q_{m}^{-1} Q_{l} Q_{m}^{-1} t^{j-i} ; q\right)_{\infty}}{\left(Q_{m_{f}} Q_{l} Q_{m}^{-1} t^{j-i+1} ; q\right)_{\infty}}, \\
Z_{\overrightarrow{\mathbf{Y}}}^{\text {fund hyper }}\left(\vec{a}, m_{f}, \epsilon_{1}, \epsilon_{2}, \beta\right) & =\prod_{l=1}^{N} \prod_{j=1}^{\infty} \frac{(\text { C. } 5)}{\left(Q_{m_{f}}^{-1} Q_{l} q t^{-j} ; q\right)_{\infty}},
\end{aligned}
$$

Another expression for the vector contribution is

$$
\begin{aligned}
Z_{\overrightarrow{\mathbf{Y}}}^{\text {vector }}\left(\vec{a}, \epsilon_{1}, \epsilon_{2}, \beta\right)= & \prod_{l, m}^{N_{c}}\left(N_{l, m}^{\overrightarrow{\mathbf{Y}}}\right)^{-1}, \\
N_{l, m}^{\overrightarrow{\mathbf{Y}}}= & \prod_{s \in Y_{l}}\left[1-e^{\left.i \beta\left[\ell_{Y_{m}}(s) \epsilon_{1}-\left(a_{Y_{l}}(s)+1\right) \epsilon_{2}+a_{l}-a_{m}\right]\right]}\right. \\
& \cdot \prod_{t \in Y_{m}}\left[1-e^{\left.i \beta\left[-\left(\ell_{Y_{l}}(t)+1\right) \epsilon_{1}+a_{Y_{m}}(t) \epsilon_{2}+a_{l}-a_{m}\right]\right]} .\right.
\end{aligned}
$$

The symbols above are defined as follows:

- $\overrightarrow{\mathbf{Y}}$ is an $N$-vector of partitions $\mathbf{Y}_{l}$. A partition is a non-increasing sequence of nonnegative integers which stabilizes at zero

$$
\mathbf{Y}_{l}=\left\{Y_{l 1} \geq Y_{l 2} \geq \ldots \geq Y_{l n_{l}+1}=0=Y_{l n_{l}+2}=Y_{l n_{l}+3}=\ldots\right\}
$$

We define

$$
\left|\mathbf{Y}_{l}\right| \equiv \sum_{i} Y_{l i}, \quad\left\|\mathbf{Y}_{l}\right\|^{2} \equiv \sum_{i} Y_{l i}^{2}, \quad|\overrightarrow{\mathbf{Y}}| \equiv \sum_{l, i} Y_{l i}
$$

The sum in (C.3) is over all such partitions. A partition $\mathbf{Y}_{l}$ can be identified with a Young diagram whose $i^{\text {th }}$ column is of height $Y_{l i}$. We denote the partition corresponding to the transposed Young diagram as $\mathbf{Y}_{l}^{t}$.

- For a box $s \in Y_{l}$ with coordinates $s=(i, j)$, we define the leg length and arm length

$$
\ell_{Y_{l}}(s) \equiv Y_{l j}^{t}-i, \quad a_{Y_{l}}(s) \equiv Y_{l i}-j .
$$


- $\vec{a}$ is an $N$-vector of deformation parameters corresponding to the equivariant action of the gauge group on the instanton moduli space. In the partition functions we compute, they are integrated over the imaginary axis and identified with the vev of a scalar field in the vector multiplet.

- $\vec{m}_{f}$ is an $N_{f}$-dimensional vector of mass deformation parameters associated to hypermultiplets. When all of the hypermultiplets are in the fundamental representation of the gauge group, $\vec{m}_{f}$ transforms as a fundamental of the flavor symmetry group $\mathrm{SU}\left(N_{f}\right)$. Mass deformations should be viewed as coming from a vev for a background vector multiplet. Physical masses are the imaginary part of this vev.

- We define

$$
q \equiv e^{i \beta \epsilon_{2}}, \quad t \equiv e^{-i \beta \epsilon_{1}}, \quad Q_{l} \equiv e^{i \beta a_{l}}, \quad Q_{m_{f}} \equiv e^{i \beta m_{f}} .
$$

This definition differs from [61] by (C.2).

- The $q$-Pochhammer symbol is defined as

$$
(x, q)_{\infty} \equiv \prod_{p=0}^{\infty}\left(1-x q^{p}\right) .
$$

- $\mathfrak{q}$ is a classical contribution equal to 20

- in $5 d$ we have

$$
\mathfrak{q}=e^{-\beta \frac{8 \pi^{2}}{g_{\mathrm{YM}}^{2}}}
$$

- In a $4 d$ calculation one uses

$$
\mathfrak{q}^{(4 d)} \equiv e^{2 \pi i \tau}
$$

where $e^{2 \pi i \tau}$ is minus the exponential of the one instanton action of the conformal theory with coupling constant

$$
\tau=\frac{\theta_{\mathrm{YM}}}{2 \pi}+\frac{4 \pi i}{g_{\mathrm{YM}}^{2}} .
$$

If the theory is not conformal, then ${ }^{21}$

$$
\mathfrak{q}^{(4 d)} \rightarrow \Lambda^{2 h^{\vee}(G)-k(R)},
$$

\footnotetext{
${ }^{20}$ Later versions of [61] include a different convention for the counting parameter, essentially redefining

$$
\mathfrak{q} \rightarrow \mathfrak{q} e^{-\frac{\epsilon_{1}+\epsilon_{2}}{2}}
$$

There is a similar factor included in the Chern-Simons contribution. We will not use these redefinitions.

${ }^{21}$ We follow [57]. $h^{\vee}(G)$ is the dual Coxeter number and $k(R)$ is the quadratic Casimir, normalized such that $k$ (adjoint) $=2 h^{\vee}$. For $\mathrm{SU}(N)$, we have $h^{\vee}=N$ and $k$ (fund) $=1$. The combination $2 h^{\vee}(G)-k(R)$ is the coefficient of the one-loop beta function for the $4 d \mathcal{N}=2$ theory with hypermultiplets in the representation $R$.
} 
where $\Lambda$ is the holomorphic dynamical scale. According to [57], the relationship between the $5 d$ and $4 d$ partition functions is

$$
\mathfrak{q}=\mathfrak{q}^{(4 d)}(-i \beta)^{2 h^{\vee}(G)-k(R)},
$$

and

$$
Z_{\text {inst }}^{(4 d)}\left(\mathfrak{q}^{(4 d)}, \vec{a}, \vec{m}_{f}, \epsilon_{1}, \epsilon_{2}\right)=\lim _{\beta \rightarrow 0} Z_{\text {inst }}\left(\mathfrak{q}^{(4 \mathrm{~d})}(-i \beta)^{2 h^{\vee}(G)-k(R)}, \vec{a}, \vec{m}_{f}, \epsilon_{1}, \epsilon_{2}, \beta\right) .
$$

- In the presence of a $5 d$ Chern-Simons term with parameter $\kappa$, we have [81]

$$
Z_{\overrightarrow{\mathbf{Y}}, \kappa}^{5 d-\mathrm{CS}}\left(\vec{a}, \epsilon_{1}, \epsilon_{2}, \beta\right)=\prod_{l=1}^{N}\left(Q_{l}^{\left|\mathbf{Y}_{l}\right|} q^{\frac{\left\|\mathbf{Y}_{l}\right\|^{2}}{2}} t^{\frac{\left\|\mathbf{Y}_{l}^{t}\right\|^{2}}{2}}\right)^{-\kappa} .
$$

An alternative version is $[61,82]$

$$
Z_{\overrightarrow{\mathbf{Y}}, \kappa}^{5 d-\mathrm{CS}}\left(\vec{a}, \epsilon_{1}, \epsilon_{2}, \beta\right)=\exp \left[i \beta \kappa \sum_{l} \sum_{(s, t) \in \mathbf{Y}_{l}}\left(a_{l}-(s-1) \epsilon_{1}-(t-1) \epsilon_{2}\right)\right] .
$$

The parameters $\epsilon_{1,2}$ are associated with the $\Omega$-deformation in the $4 d$ theory. They take the following values:

- in the derivation of the prepotential for the Seiberg-Witten solution one takes

$$
\epsilon_{1}=-\epsilon_{2} \equiv i \hbar, \quad \beta \rightarrow 0,
$$

eventually extracting the leading piece at $\hbar \rightarrow 0$.

- in the computation on the squashed four-sphere, with squashing parameters $\omega_{1}=$ $\ell^{-1}, \omega_{2}=\tilde{\ell}^{-1}$, the instanton contribution from the north pole involves

$$
\epsilon_{1}=\omega_{1}, \quad \epsilon_{2}=\omega_{2},
$$

and for anti-instantons from the south pole

$$
\epsilon_{1}=\omega_{2}, \quad \epsilon_{2}=\omega_{1},
$$

and we take the limit $\beta \rightarrow 0$. For the squashed $\mathbb{S}^{4}$ corresponding to the $n^{\text {th }}$ supersymmetric Rényi entropy, we choose

$$
\omega_{1}=1, \quad \omega_{2}=\frac{1}{n} .
$$

- in the computation on the five-sphere, $\beta$ is the circumference of the circle fiber and there are three fixed points, the values at which are given in table 1 . These correspond to the values given in $[50,63]$.

For the squashed $\mathbb{S}^{5}$ corresponding to the $n^{\text {th }}$ supersymmetric Rényi entropy, we choose

$$
\left(\omega_{1}, \omega_{2}, \omega_{3}\right)=\left(1,1, \frac{1}{n}\right)
$$




\begin{tabular}{|c|c|c|c|c|c|}
\hline$\#$ & $\beta$ & $\epsilon_{1}$ & $\epsilon_{2}$ & $q$ & $t$ \\
\hline 1 & $\frac{2 \pi}{\omega_{1}}$ & $\omega_{2}$ & $\omega_{3}$ & $e^{2 \pi i \frac{\omega_{3}}{\omega_{1}}}$ & $e^{-2 \pi i \frac{\omega_{2}}{\omega_{1}}}$ \\
\hline 2 & $\frac{2 \pi}{\omega_{2}}$ & $\omega_{3}$ & $\omega_{1}$ & $e^{2 \pi i \frac{\omega_{1}}{\omega_{2}}}$ & $e^{-2 \pi i \frac{\omega_{3}}{\omega_{2}}}$ \\
\hline 3 & $\frac{2 \pi}{\omega_{3}}$ & $\omega_{1}$ & $\omega_{2}$ & $e^{2 \pi i \frac{\omega_{2}}{\omega_{3}}}$ & $e^{-2 \pi i \frac{\omega_{1}}{\omega_{3}}}$ \\
\hline
\end{tabular}

Table 1. Parameters entering the $q$-deformed Nekrasov partition function at three fixed points on a squashed $\mathbb{S}^{5}$.

Open Access. This article is distributed under the terms of the Creative Commons Attribution License (CC-BY 4.0), which permits any use, distribution and reproduction in any medium, provided the original author(s) and source are credited.

\section{References}

[1] P. Calabrese and J.L. Cardy, Entanglement entropy and quantum field theory, J. Stat. Mech. 0406 (2004) P06002 [hep-th/0405152] [INSPIRE].

[2] H. Casini and M. Huerta, Entanglement entropy for the n-sphere, Phys. Lett. B 694 (2011) 167 [arXiv:1007.1813] [INSPIRE].

[3] I.R. Klebanov, S.S. Pufu, S. Sachdev and B.R. Safdi, Rényi Entropies for Free Field Theories, JHEP 04 (2012) 074 [arXiv:1111.6290] [InSPIRE].

[4] D.V. Fursaev and S.N. Solodukhin, On the description of the Riemannian geometry in the presence of conical defects, Phys. Rev. D 52 (1995) 2133 [hep-th/9501127] [INSPIRE].

[5] L.-Y. Hung, R.C. Myers, M. Smolkin and A. Yale, Holographic Calculations of Rényi Entropy, JHEP 12 (2011) 047 [arXiv:1110.1084] [INSPIRE].

[6] T. Nishioka and I. Yaakov, Supersymmetric Rényi Entropy, JHEP 10 (2013) 155 [arXiv: 1306.2958] [INSPIRE].

[7] H. Casini, M. Huerta and R.C. Myers, Towards a derivation of holographic entanglement entropy, JHEP 05 (2011) 036 [arXiv: 1102.0440] [INSPIRE].

[8] L. Bianchi, M. Meineri, R.C. Myers and M. Smolkin, Rényi entropy and conformal defects, JHEP 07 (2016) 076 [arXiv: 1511.06713] [INSPIRE].

[9] H. Casini and M. Huerta, Entanglement entropy in free quantum field theory, J. Phys. A 42 (2009) 504007 [arXiv:0905. 2562] [INSPIRE].

[10] X. Huang, S.-J. Rey and Y. Zhou, Three-dimensional SCFT on conic space as hologram of charged topological black hole, JHEP 03 (2014) 127 [arXiv:1401.5421] [INSPIRE].

[11] T. Nishioka, The Gravity Dual of Supersymmetric Rényi Entropy, JHEP 07 (2014) 061 [arXiv: 1401.6764] [INSPIRE].

[12] M. Crossley, E. Dyer and J. Sonner, Super-Rényi entropy \&3 Wilson loops for $\mathcal{N}=4 S Y M$ and their gravity duals, JHEP 12 (2014) 001 [arXiv:1409.0542] [INSPIRE].

[13] X. Huang and Y. Zhou, $\mathcal{N}=4$ super-Yang-Mills on conic space as hologram of $S T U$ topological black hole, JHEP 02 (2015) 068 [arXiv:1408.3393] [INSPIRE]. 
[14] N. Hama, T. Nishioka and T. Ugajin, Supersymmetric Rényi entropy in five dimensions, JHEP 12 (2014) 048 [arXiv: 1410.2206] [INSPIRE].

[15] L.F. Alday, P. Richmond and J. Sparks, The holographic supersymmetric Rényi entropy in five dimensions, JHEP 02 (2015) 102 [arXiv:1410.0899] [INSPIRE].

[16] A. Giveon and D. Kutasov, Supersymmetric Rényi entropy in $\mathrm{CFT}_{2}$ and $A d S_{3}$, JHEP 01 (2016) 042 [arXiv: 1510.08872] [INSPIRE].

[17] H. Mori, Supersymmetric Rényi entropy in two dimensions, JHEP 03 (2016) 058 [arXiv: 1512.02829] [INSPIRE].

[18] J. Nian and Y. Zhou, Rényi entropy of a free $(2,0)$ tensor multiplet and its supersymmetric counterpart, Phys. Rev. D 93 (2016) 125010 [arXiv:1511.00313] [INSPIRE].

[19] C. Closset, T.T. Dumitrescu, G. Festuccia and Z. Komargodski, The Geometry of Supersymmetric Partition Functions, JHEP 01 (2014) 124 [arXiv: 1309.5876] [INSPIRE].

[20] A. Kapustin and N. Seiberg, Coupling a QFT to a TQFT and Duality, JHEP 04 (2014) 001 [arXiv: 1401.0740] [INSPIRE].

[21] R. Brooks and S.J. Gates, Jr., Extended supersymmetry and superBF gauge theories, Nucl. Phys. B 432 (1994) 205 [hep-th/9407147] [INSPIRE].

[22] D. Gaiotto and E. Witten, S-duality of Boundary Conditions In $N=4$ Super Yang-Mills Theory, Adv. Theor. Math. Phys. 13 (2009) 721 [arXiv:0807.3720] [InSPIRE].

[23] G.W. Moore and N. Seiberg, Taming the Conformal Zoo, Phys. Lett. B 220 (1989) 422 [INSPIRE].

[24] A. Kapustin, B. Willett and I. Yaakov, Exact results for supersymmetric abelian vortex loops in 2+1 dimensions, JHEP 06 (2013) 099 [arXiv:1211.2861] [INSPIRE].

[25] N. Drukker, T. Okuda and F. Passerini, Exact results for vortex loop operators in $3 d$ supersymmetric theories, JHEP 07 (2014) 137 [arXiv:1211.3409] [INSPIRE].

[26] A. Kapustin, B. Willett and I. Yaakov, Exact Results for Wilson Loops in Superconformal Chern-Simons Theories with Matter, JHEP 03 (2010) 089 [arXiv:0909.4559] [INSPIRE].

[27] D.L. Jafferis, The Exact Superconformal R-Symmetry Extremizes Z, JHEP 05 (2012) 159 [arXiv: 1012.3210] [INSPIRE].

[28] V. Pestun, Localization of gauge theory on a four-sphere and supersymmetric Wilson loops, Commun. Math. Phys. 313 (2012) 71 [arXiv:0712.2824] [INSPIRE].

[29] N. Hama and K. Hosomichi, Seiberg-Witten Theories on Ellipsoids, JHEP 09 (2012) 033 [arXiv: 1206.6359] [INSPIRE].

[30] B. de Wit and F. Saueressig, Off-shell $N=2$ tensor supermultiplets, JHEP 09 (2006) 062 [hep-th/0606148] [INSPIRE].

[31] D.Z. Freedman and A. Van Proeyen, Supergravity, Cambridge University Press, (2012).

[32] V. Pestun, Localization for $\mathcal{N}=2$ Supersymmetric Gauge Theories in Four Dimensions, in New Dualities of Supersymmetric Gauge Theories, J. Teschner ed., Springer, (2016), pp. 159-194, arXiv:1412.7134.

[33] T. Kugo and K. Ohashi, Supergravity tensor calculus in $5-D$ from 6-D, Prog. Theor. Phys. 104 (2000) 835 [hep-ph/0006231] [INSPIRE]. 
[34] K. Hosomichi, R.-K. Seong and S. Terashima, Supersymmetric Gauge Theories on the Five-Sphere, Nucl. Phys. B 865 (2012) 376 [arXiv:1203.0371] [InSPIRE].

[35] S. Gukov and E. Witten, Gauge Theory, Ramification, And The Geometric Langlands Program, hep-th/0612073 [INSPIRE].

[36] S. Gukov, Surface Operators, in New Dualities of Supersymmetric Gauge Theories,

J. Teschner ed., Springer, (2016), pp. 223-259, arXiv:1412.7127.

[37] T. Okuda and V. Pestun, On the instantons and the hypermultiplet mass of $N=2^{*}$ super Yang-Mills on $S^{4}$, JHEP 03 (2012) 017 [arXiv:1004.1222] [INSPIRE].

[38] K. Hosomichi, N=2 SUSY gauge theories on $S^{4}$, J. Phys. A 50 (2017) 443010 [arXiv: 1608.02962] [INSPIRE].

[39] H.-C. Kim, S. Kim, E. Koh, K. Lee and S. Lee, On instantons as Kaluza-Klein modes of M5-branes, JHEP 12 (2011) 031 [arXiv:1110.2175] [INSPIRE].

[40] M. Bullimore and H.-C. Kim, The Superconformal Index of the (2,0) Theory with Defects, JHEP 05 (2015) 048 [arXiv: 1412.3872] [INSPIRE].

[41] N. Hama, K. Hosomichi and S. Lee, SUSY Gauge Theories on Squashed Three-Spheres, JHEP 05 (2011) 014 [arXiv: 1102.4716] [INSPIRE].

[42] Y. Imamura and D. Yokoyama, $\mathcal{N}=2$ supersymmetric theories on squashed three-sphere, Phys. Rev. D 85 (2012) 025015 [arXiv:1109.4734] [INSPIRE].

[43] J. Källén and M. Zabzine, Twisted supersymmetric $5 D$ Yang-Mills theory and contact geometry, JHEP 05 (2012) 125 [arXiv:1202.1956] [INSPIRE].

[44] Y. Imamura, Supersymmetric theories on squashed five-sphere, PTEP 2013 (2013) 013B04 [arXiv: 1209.0561] [INSPIRE].

[45] H.-C. Kim, J. Kim and S. Kim, Instantons on the 5-sphere and M5-branes, arXiv: 1211.0144 [INSPIRE].

[46] Y. Imamura, Perturbative partition function for squashed $S^{5}$, PTEP 2013 (2013) 073B01 [arXiv: 1210.6308] [INSPIRE].

[47] J. Källén, J. Qiu and M. Zabzine, The perturbative partition function of supersymmetric $5 D$ Yang-Mills theory with matter on the five-sphere, JHEP 08 (2012) 157 [arXiv:1206.6008] [INSPIRE].

[48] G. Lockhart and C. Vafa, Superconformal Partition Functions and Non-perturbative Topological Strings, arXiv:1210.5909 [INSPIRE].

[49] H.-C. Kim and S. Kim, M5-branes from gauge theories on the 5-sphere, JHEP 05 (2013) 144 [arXiv:1206.6339] [INSPIRE].

[50] S. Pasquetti, Holomorphic blocks and the 5d AGT correspondence, J. Phys. A 50 (2017) 443016 [arXiv: 1608.02968] [INSPIRE].

[51] N. Kurokawa et al., Derivatives of multiple sine functions, Proc. Japan Acad. A 80 (2004) 65-69.

[52] N.A. Nekrasov, Seiberg-Witten prepotential from instanton counting, Adv. Theor. Math. Phys. 7 (2003) 831 [hep-th/0206161] [INSPIRE].

[53] N. Nekrasov and A. Okounkov, Seiberg-Witten theory and random partitions, Prog. Math. 244 (2006) 525 [hep-th/0306238] [INSPIRE]. 
[54] F. Fucito, J.F. Morales and R. Poghossian, Multi instanton calculus on ALE spaces, Nucl. Phys. B 703 (2004) 518 [hep-th/0406243] [INSPIRE].

[55] H. Kanno and Y. Tachikawa, Instanton counting with a surface operator and the chain-saw quiver, JHEP 06 (2011) 119 [arXiv:1105.0357] [INSPIRE].

[56] N. Wyllard, Instanton partition functions in $N=2 \mathrm{SU}(N)$ gauge theories with a general surface operator and their W-algebra duals, JHEP 02 (2011) 114 [arXiv:1012.1355] [INSPIRE].

[57] Y. Tachikawa, A Review on Instanton Counting and W-Algebras, in New Dualities of Supersymmetric Gauge Theories, J. Teschner ed., Springer, (2016), pp. 79-120, arXiv: 1412.7121.

[58] S. Kim, K.-M. Lee and S. Lee, Dyonic Instantons in 5-dim Yang-Mills Chern-Simons Theories, JHEP 08 (2008) 064 [arXiv:0804.1207] [INSPIRE].

[59] B. Collie and D. Tong, Instantons, Fermions and Chern-Simons Terms, JHEP 07 (2008) 015 [arXiv: 0804.1772] [INSPIRE].

[60] H.-C. Kim, S.-S. Kim and K. Lee, 5-dim Superconformal Index with Enhanced $E_{n}$ Global Symmetry, JHEP 10 (2012) 142 [arXiv:1206.6781] [INSPIRE].

[61] H. Awata and H. Kanno, Refined BPS state counting from Nekrasov's formula and Macdonald functions, Int. J. Mod. Phys. A 24 (2009) 2253 [arXiv:0805.0191] [InSPIRE].

[62] P. Bueno, R.C. Myers and W. Witczak-Krempa, Universal corner entanglement from twist operators, JHEP 09 (2015) 091 [arXiv: 1507.06997] [INSPIRE].

[63] F. Nieri, S. Pasquetti, F. Passerini and A. Torrielli, 5D partition functions, q-Virasoro systems and integrable spin-chains, JHEP 12 (2014) 040 [arXiv:1312.1294] [INSPIRE].

[64] F. Nieri, S. Pasquetti and F. Passerini, $3 d$ and $5 d$ Gauge Theory Partition Functions as q-deformed CFT Correlators, Lett. Math. Phys. 105 (2015) 109 [arXiv:1303.2626] [INSPIRE].

[65] G. Festuccia, J. Qiu, J. Winding and M. Zabzine, $\mathcal{N}=2$ supersymmetric gauge theory on connected sums of $S^{2} \times S^{2}$, JHEP 03 (2017) 026 [arXiv: 1611.04868] [INSPIRE].

[66] J. Qiu, L. Tizzano, J. Winding and M. Zabzine, Modular properties of full 5D SYM partition function, JHEP 03 (2016) 193 [arXiv:1511.06304] [INSPIRE].

[67] S. Ryu and T. Takayanagi, Holographic derivation of entanglement entropy from AdS/CFT, Phys. Rev. Lett. 96 (2006) 181602 [hep-th/0603001] [INSPIRE].

[68] S. Ryu and T. Takayanagi, Aspects of Holographic Entanglement Entropy, JHEP 08 (2006) 045 [hep-th/0605073] [INSPIRE].

[69] T. Faulkner, A. Lewkowycz and J. Maldacena, Quantum corrections to holographic entanglement entropy, JHEP 11 (2013) 074 [arXiv:1307.2892] [INSPIRE].

[70] T. Barrella, X. Dong, S.A. Hartnoll and V.L. Martin, Holographic entanglement beyond classical gravity, JHEP 09 (2013) 109 [arXiv: 1306.4682] [INSPIRE].

[71] X. Dong, The Gravity Dual of Rényi Entropy, Nature Commun. 7 (2016) 12472 [arXiv: 1601.06788] [INSPIRE].

[72] J.M. Maldacena, Wilson loops in large- $N$ field theories, Phys. Rev. Lett. 80 (1998) 4859 [hep-th/9803002] [INSPIRE]. 
[73] S.-J. Rey and J.-T. Yee, Macroscopic strings as heavy quarks in large- $N$ gauge theory and anti-de Sitter supergravity, Eur. Phys. J. C 22 (2001) 379 [hep-th/9803001] [InSPIRE].

[74] N. Drukker, D.J. Gross and H. Ooguri, Wilson loops and minimal surfaces, Phys. Rev. D 60 (1999) 125006 [hep-th/9904191] [INSPIRE].

[75] N.R. Constable, J. Erdmenger, Z. Guralnik and I. Kirsch, Intersecting D-3 branes and holography, Phys. Rev. D 68 (2003) 106007 [hep-th/0211222] [INSPIRE].

[76] J. Gomis and S. Matsuura, Bubbling surface operators and S-duality, JHEP 06 (2007) 025 [arXiv: 0704.1657] [INSPIRE].

[77] N. Drukker, J. Gomis and S. Matsuura, Probing N $=4$ SYM With Surface Operators, JHEP 10 (2008) 048 [arXiv: 0805.4199] [INSPIRE].

[78] E. Koh and S. Yamaguchi, Holography of BPS surface operators, JHEP 02 (2009) 012 [arXiv:0812.1420] [INSPIRE].

[79] N. Kurokawa and S.-y. Koyama, Multiple sine functions, Forum Mathematicum 15 (2003) 839.

[80] A.B. Zamolodchikov and A.B. Zamolodchikov, Structure constants and conformal bootstrap in Liouville field theory, Nucl. Phys. B 477 (1996) 577 [hep-th/9506136] [INSPIRE].

[81] P. Sulkowski, Matrix models for beta-ensembles from Nekrasov partition functions, JHEP 04 (2010) 063 [arXiv:0912.5476] [INSPIRE].

[82] Y. Tachikawa, Five-dimensional Chern-Simons terms and Nekrasov's instanton counting, JHEP 02 (2004) 050 [hep-th/0401184] [INSPIRE]. 